\title{
La educación y la distribución del ingreso en el Brasil urbano, 1976-1996
}

\section{Francisco H. G. Ferreira}

Profesor, Departamento

de Economía,

Pontificia Universidad Católica

de Rio de Janeiro

fferreira@econ.puc-rio.br

\section{Ricardo Paes de Barros}

Instituto de Investigación

Económica Aplicada,

Rio de Janeiro

rbarros@ipea.gov.br
Pese a la enorme inestabilidad macroeconómica, a primera vista las distribuciones del ingreso urbano en el Brasil en 1976 y 1996 se ven muy parecidas. El ingreso medio de los hogares por habitante estaba estacionario, con un crecimiento minúsculo de $4.3 \%$ acumulado en los dos decenios. El coeficiente de Gini oscilaba apenas sobre el 0.59 en ambos años y la incidencia de la pobreza (con respecto a una línea de pobreza de 60 reales al mes a precios de 1996) permanecía de hecho inalterada, en $22 \%$. Sin embargo, bajo esta estabilidad aparente operaba una poderosa conjunción del mercado laboral y la dinámica demográfica y educativa para generar, como uno de sus efectos, un aumento considerable de la pobreza extrema urbana. Usando una metodología de descomposición basada en la microsimulación, que aplica como factores endógenos el ingreso del trabajo, las opciones laborales individuales y las decisiones en materia de educación, demostramos que influyeron en la distribución del ingreso, por una parte, el descenso en el rendimiento medio de la educación y la experiencia, así como cambios pauperizantes en la estructura de las ocupaciones y la participación de la fuerza de trabajo (todo lo cual tendió a aumentar la pobreza), y, por la otra, el aumento en el nivel de los logros educativos en toda la distribución y la reducción progresiva de las tasas de dependencia (factores ambos que tendieron a disminuir la pobreza). 


\section{I}

\section{Introducción}

Los dos decenios entre 1974 y 1994 —entre la primera crisis petrolera y la vuelta a la estabilidad con el Plan Real- fueron tristes para el Brasil, tanto si se les compara con el crecimiento del país durante los años del "milagro brasileño" de 1968-1973, como si el parangón se hace con los otros principales países en desarrollo, principalmente los del Asia, en períodos posteriores. Ante todo, se caracterizaron por desequilibrios macroeconómicos persistentes, cuyos síntomas principales eran una inflación irreductible — alta y aceleradajunto con una evolución temporal del producto interno bruto caracterizada por una volatilidad extraordinaria y una tendencia muy baja al crecimiento.

Las crisis macroeconómicas originaron tres congelamientos de precios y salarios (el Plan Cruzado de 1986, el Plan Bresser de 1987 y el Plan Verano de 1989), seguidos todos ellos por tasas más altas de inflación, un congelamiento temporal de activos financieros (el plan Collor de 1990) y por último una reforma monetaria con éxito seguida de la adopción de un ancla nominal en 1994 (el Plan Real). La moneda nacional fue rebautizada cuatro veces. ${ }^{1}$ En todo el período la política macroeconómica casi sin excepción se caracterizó por una política fiscal relativamente relajada con una creciente severidad monetaria.

Además, estaban ocurriendo grandes cambios estructurales. La población del Brasil aumentó en $46.6 \%$ entre 1976 y 1996, con un mayor grado de urbanización (la tasa se elevó de $68 \%$ a $77 \%$ ). El grado medio de instrucción de la población de 10 años y más

\footnotetext{
$\square$ Esta es una versión resumida de "The slippery slope: Explaining the increase in extreme poverty in urban Brazil, 1976-1996", publicado en The Brazilian Review of Econometrics, en 1999. Agradecemos la orientación y apoyo de François Bourguignon y los comentarios de James Heckman, Nora Lustig, Naércio Menezes Filho, y de participantes en la Conferencia LACEA celebrada en 1998 en Buenos Aires, la conferencia AEA 2000 celebrada en Boston, y un seminario en la Universidad de Cornell. También agradecemos el apoyo financiero del Banco Mundial. Nuestra mayor gratitud la debemos a Phillipe George Leite, Roberta Barreto, Carlos Henrique Corseuil, Sérgio Fippo, Luis Eduardo Guedes, Cristiana Lopes, Vanessa Moreira, Daniele Reis y Alinne Veiga por su magnífica asistencia en la investigación.

${ }^{1}$ Se cambió el crucero por el cruzado en 1986; el cruzado por el nuevo cruzado en 1989; el nuevo cruzado por el crucero en 1990 y el crucero por el real en 1994.
}

subió de 3.2 años a 5.3 años de escolaridad efectiva. ${ }^{2}$ El desempleo abierto llegó a generalizarse cada vez más. La composición sectorial de la fuerza de trabajo cambió: se abandonaron la agricultura y la manufactura para ingresar en los servicios. Bajó considerablemente el grado de formalidad de la fuerza de trabajo: la proporción de trabajadores documentados (asalariados con documentación formal) decayó casi a la mitad, de poco menos del $60 \%$ a poco más del $30 \%$ de los trabajadores. Y, sin embargo, pese a la crisis macroeconómica y los continuos cambios estructurales, una ojeada a los principales indicadores de la incidencia de la desigualdad y la pobreza que aparecen en el cuadro 1 parecería mostrar que la distribución de los ingresos urbanos en el Brasil apenas si cambió entre 1976 y 1996.

Sin embargo, como sucede a menudo, lo que se aprecia a primera vista puede ser engañoso. Esta aparente estabilidad de la distribución encubre un número de fuertes variaciones, a menudo contrapuestas, en cuatro aspectos: la rentabilidad de la educación en los mercados del trabajo; la distribución de los logros educativos entre la población; la composición de las opciones ocupacionales, y la estructura demográfica derivada de las opciones sobre fecundidad de los hogares. En el presente estudio señalamos dos enigmas en la evolución reciente de la distribución del ingreso urbano en el Brasil en el período 1976-1996 y sugerimos algunas explicaciones.

El primer enigma radica en la combinación de un crecimiento (lento) de los ingresos medios o una desigualdad que desciende ligeramente, por un lado, con el aumento de la extrema pobreza, por el otro. Sostenemos que esta contradicción sólo puede explicarse por el aumento del número de hogares muy pobres, que parecen estar del todo excluidos de los mercados del trabajo y de las redes de seguridad oficiales. Este grupo está atrapado en la indigencia al fondo de la distribución de los ingresos urbanos del Brasil y contribuye al alza de las mediciones de la pobreza que son

\footnotetext{
${ }^{2}$ Los años "efectivos" de educación corresponden al último curso terminado, y no cuentan las repeticiones. Todas las cifras se tomaron de Ferreira y Paes de Barros (1999).
} 


\begin{tabular}{|c|c|c|c|c|}
\hline & 1976 & 1981 & 1985 & 1996 \\
\hline PIB per cápita anual (en reales a precios constantes) & 4040 & 4442 & 4540 & 4945 \\
\hline Tasa anual de inflación ${ }^{\mathrm{a}}$ & $42 \%$ & $84 \%$ & $190 \%$ & $9 \%$ \\
\hline Desempleo abierto ${ }^{\mathrm{b}}$ & $1.82 \%$ & $4.26 \%$ & $3.38 \%$ & $6.95 \%$ \\
\hline Educación (promedio de años) ${ }^{\text {cd }}$ & 3.23 & 4.01 & 4.36 & 5.32 \\
\hline Tasa de urbanización ${ }^{\mathrm{d}}$ & $67.8 \%$ & $77.3 \%$ & $77.3 \%$ & $77.0 \%$ \\
\hline Trabajadores por cuenta propia como proporción de la fuerza de trabajo ${ }^{\mathrm{d}}$ & $27.03 \%$ & $26.20 \%$ & $26.19 \%$ & $27.21 \%$ \\
\hline Ingreso medio por habitante de los hogares urbanos ${ }^{\mathrm{d}}$ & 265.10 & 239.08 & 243.15 & 276.46 \\
\hline Desigualdad (coeficiente de Gini) ${ }^{\mathrm{d}}$ & 0.595 & 0.561 & 0.576 & 0.591 \\
\hline Desigualdad (índice Theil $-\mathrm{T})^{\mathrm{d}}$ & 0.760 & 0.610 & 0.657 & 0.694 \\
\hline Incidencia de la pobreza $(\mathrm{R} \$ 30 / \mathrm{mes})^{\mathrm{f}}$ & 0.0681 & 0.0727 & 0.0758 & 0.0922 \\
\hline Incidencia de la pobreza $(\mathrm{R} \$ 60 / \mathrm{mes})^{\mathrm{f}}$ & 0.2209 & 0.2149 & 0.2274 & 0.2176 \\
\hline
\end{tabular}

a Por ciento de enero a diciembre. Basado en IGP-DI para 1976 y el INPC-R para los demás años.

b Basado en el índice de cesantía metropolitana del Instituto Brasileño de Geografía y Estadística.

c Para todos los individuos de 10 años y más, en zonas urbanas.

d Calculado por los autores sobre la base de muestras de la encuesta nacional de hogares (PNAD).

e Definido como la proporción de empleados documentados en el total de los asalariados y trabajadores por cuenta propia.

f Urbano solamente, por mes y deflacionado espacialmente. Expresado en reales constantes de 1996.

particularmente sensibles a la profundidad $-\mathrm{P}(1)-\mathrm{y}$ la severidad - $\mathrm{P}(2)$ - de la pobreza, en particular cuando ésta se define por la línea más baja de ingresos. ${ }^{3}$ Esto se observa crudamente en la sección II, donde el gráfico 1 ilustra los "desfiles de Pen" (truncados) para los cuatro años estudiados. ${ }^{4} \mathrm{El}$ principal canal endógeno por el cual se capta la marginalización de este grupo en nuestro modelo es el cambio de sus "decisiones" ocupacionales, con abandono del trabajo asalariado o por cuenta propia e ingreso a la cesantía o salida de la fuerza de trabajo.

Los hechos que examinaremos en la sección IV revelan un descenso del perfil ingreso-enseñanza, controlado por edad y género, tanto en el sector asalariado como el de trabajo por cuenta propia. ${ }^{5}$ Pese a que el perfil se volvió ligeramente convexo, la magnitud del cambio supone un descenso en la rentabilidad (media) de la educación para todos los niveles impor-

\footnotetext{
${ }^{3}$ Todas las medidas de la pobreza señaladas en este estudio son de la clase $P(\alpha)$ de las medidas descomponibles ideadas por Foster, Greer y Thorbecke (1984). Un aumento de $\alpha$ implica un incremento en la ponderación que representa la distancia entre los ingresos de las personas y la línea de pobreza.

${ }^{4}$ La noción de "desfiles" de perceptores de ingresos fue ideada por Jan Pen. Los "desfiles de Pen" son la inversa matemática de las funciones de distribución; es decir, grafican los ingresos percibidos por cada persona (o grupos de personas) cuando se ordenan según su ingreso.

${ }^{5}$ Esta evolución se da entre 1976 y 1996, con una elevación en el decenio de 1980 (véase más adelante el gráfico 4).
}

tantes de la enseñanza. Así también, la rentabilidad media de la experiencia laboral decayó sin excepción desde los 0 hasta los 50 años de experiencia (como veremos más adelante en el gráfico 5). El efecto conjunto de estos cambios —el efecto precio- fue el aumento de la pobreza simulada para todas las mediciones y ambas líneas de pobreza. La desigualdad simulada también subió, pero mucho menos. Ambos efectos se exageran cuando también se toman en cuenta los cambios (hasta 1996) en los factores determinantes de las decisiones de participación en la fuerza de trabajo.

El segundo enigma es entonces cuáles son las fuerzas que contrarrestan estos efectos de precio y elección ocupacional como para que se explique la estabilidad observada de la desigualdad y la pobreza de la "línea superior". 6 Descubrimos que se trataba esencialmente de una combinación de un mejor nivel educativo, que hacía subir a los trabajadores por la cuesta menos escarpada de ingreso-educación, con un aumento de la correlación entre el ingreso familiar y el tamaño de la familia, provocado por una reducción más que proporcional en las tasas de dependencia y el tamaño de la familia para los estratos pobres. Este factor demográfico tenía un efecto directo sobre el ingre-

\footnotetext{
${ }^{6}$ Pobreza de la línea superior significa en este trabajo la incidencia de la pobreza con respecto a la línea de pobreza de 60 reales al mes.
} 
so por habitante —al reducirse el denominador - y efectos indirectos, por decisiones de participación e ingresos más elevados.

Intentamos resolver estos problemas con una descomposición de los cambios distribucionales basada en la microsimulación, según el método desarrollado por Bourguignon, Ferreira y Lustig (1998), que a su vez elabora sobre los trabajos de Almeida dos Reis y Paes de Barros (1991) y Juhn, Murphy y Pierce (1993). El método tiene dos características sobresalientes. En primer lugar, a diferencia de otras descomposiciones dinámicas de la desigualdad, como la propuesta por Mookherjee y Shorrocks (1982), descompone los efectos de cambios en toda una distribución, en vez de hacerlo en una estadística escalar sumaria (como la desviación logarítmica media). Ello permite una mayor versatilidad: con un mismo marco, pueden efectuarse un sinnúmero de simulaciones para investigar los efectos de cambios en parámetros específicos de cualquier número de cuantificadores de la desigualdad o la pobreza (y a continuación para cualquier cantidad de líneas de pobreza o hipótesis sobre las escalas de equivalencia). En segundo lugar, la distribución dinámica que el método descompone es la distribución de ingresos per cápita de los hogares, pero con los indi- viduos como unidades receptoras. Por lo tanto, a diferencia de los estudios centrados exclusivamente en el mercado del trabajo, se toma explícitamente en cuenta la influencia de la composición de los hogares sobre las condiciones de vida y las decisiones de participación. Se dio el caso que estos factores son de gran importancia para comprender mejor la dinámica del fenómeno en estudio.

En resumen, el artículo se organiza de la manera siguiente. La sección II resume las conclusiones principales que emanan de las publicaciones sobre la distribución del ingreso en el Brasil durante el período examinado y presenta estadísticas resumidas y comparaciones de dominancia sobre las cuatro distribuciones observadas que se analizan en el estudio, a saber, 1976, 1981, 1985 y 1996. La sección III explica someramente cómo se adaptó el modelo de Bourguignon, Ferreira y Lustig (1998) al caso del Brasil. En la sección IV se muestran los resultados de la etapa de estimaciones y se analizan algunas de sus implicaciones. En la sección $\mathrm{V}$ se describen los principales resultados de la etapa de simulación y se descomponen los cambios observados en la pobreza y la desigualdad. En la sección VI se presentan las conclusiones y se señalan algunas repercusiones de política.

\section{II}

\section{La distribución del ingreso en el Brasil entre 1976 y 1996: somero examen de la literatura sobre el tema y de nuestras series de datos}

En la literatura examinada hay consenso en general sobre las grandes tendencias de la desigualdad en el Brasil desde los primeros tiempos en que hubo estadísticas razonables, el decenio de 1960. El coeficiente de Gini subió considerablemente en ese decenio, desde alrededor de 0.500 en 1960 a 0.565 en 1970 (véase Bonelli y Sedlacek, 1989). Hubo diferentes corrientes de opinión sobre las causas de este aumento, encabezadas principalmente por Albert Fishlow, de un lado, y Carlos Langoni, del otro, con un acuerdo general de que en los años sesenta aumentó mucho la dispersión en la distribución del ingreso brasileño. ${ }^{7}$

\footnotetext{
${ }^{7}$ La controversia entre Fishlow y Langoni giraba en torno a la importancia de la educación frente a las políticas represivas del
}

En el decenio de 1970 hubo una evolución más compleja. La desigualdad del ingreso aumentó entre 1970 y 1976, llegó a un máximo en ese año y luego disminuyó entre 1977 y 1981, tanto para la distribución de los ingresos individuales totales en la población económicamente activa (PEA) como para la distribución completa de los ingresos per cápita de la unidad familiar (véase Bonelli y Sedlacek, 1989; Hoffman, 1989; Ramos, 1993). El año de crisis de 1981 marcó un mínimo local en la serie de la desigualdad, se apliquen

mercado de trabajo en la determinación del alto nivel de desigualdad. Véase, por ejemplo, Fishlow (1972), Langoni (1973) y Bacha y Taylor (1980). 
el coeficiente de Gini o el índice Theil-T. A partir de 1981, ésta aumentó en los años depresivos de 1982 y 1983. Algunos autores señalan el pequeño descenso de algunos índices en 1984, pero en 1985 continuó el ascenso. En 1986, el año del Plan Cruzado, se interrumpió la serie por efecto tanto de una brusca (aunque transitoria) baja de la inflación, como de un gran aumento de los ingresos declarados por los hogares. La estabilidad y el crecimiento económico se tradujeron en un descenso de la desigualdad registrada, según todos los autores. De ahí en adelante, al fracasar el intento de estabilización que representaba el Plan Cruzado y volver la inflación con estancamiento, la desigualdad siguió en su tendencia ascendente, con el coeficiente de Gini en 0.606 al final del decenio.

Las tendencias generales que señala la literatura se reflejan en las estadísticas para los años que interesan especialmente en este estudio, a saber, 1976, 1981, 1985 y 1996. Las distribuciones para cada uno de esos años corresponden a las de la encuesta nacional de hogares (PNAD) que realiza el Instituto Brasileño de Geografía y Estadística (IBGE). Salvo donde se indique expresamente lo contrario, nos ocuparemos sólo de las áreas urbanas, siendo el concepto de bienestar equivalente al total de ingresos per cápita del hogar (en reales constantes, ajustados por diferencias regionales en el nivel de vida medio) y la unidad de análisis es el individuo. Los pormenores sobre la cobertura de la muestra y la metodología de la PNAD, así como el tamaño de la muestra, la definición de las variables claves del ingreso, los problemas de deflación espacial y temporal, y los reajustes con respecto a la base de las cuentas nacionales se describen en Ferreira y Paes de Barros (1999).

El cuadro 2 presenta varias estadísticas sintéticas sobre estas distribuciones, aparte de la media que aparece en el cuadro 1. Los cuatro índices de desigualdad que se emplearán en todo este trabajo son el coeficiente de Gini y tres miembros de la Clase de Entropía Generalizada de índices de desigualdad, $E(\Phi)$. Específicamente, hemos escogido $E(0)$, conocido también como la desviación media logarítmica o índice Theil-L; $E$ (1) llamado comúnmente índice Theil-T, y $E(2)$, que es la mitad del cuadrado del coeficiente de variación. Estos índices proporcionan un rango útil de sensibilidad para distintas partes de la distribución. $E(0)$ es un indicador más sensible para la porción inferior de la distribución, en tanto que $E(2)$ resulta más fiel para los ingresos superiores. $E(1)$ queda entre los dos, en tanto que el coeficiente de Gini da mayor ponderación alrededor de la media.
Presentamos también tres índices de pobreza de la clase descomponible aditivamente: $P(\alpha)$. $P(0)$, conocido también como índice de recuento (headcount), mide la incidencia de la pobreza; $P(1)$ es el déficit de pobreza normalizado y $P(2)$ es un promedio de déficit cuadrados normalizados, con lo cual se da mayor ponderación a los ingresos más alejados de la línea de pobreza. Calculamos cada uno de ellos con relación a dos líneas de pobreza, que corresponden repectivamente a ingresos de uno y de dos reales por día, a precios de $1996 .^{8}$

Cada uno de estos índices de pobreza y desigualdad se dan tanto para la distribución de los ingresos totales del hogar per cápita como para la distribución igualada empleando la clase paramétrica de escalas equivalentes (en que $\theta=0.5$ ) de Buhmann, Rainwater, Schmaus y Smeeding (1988). Este sistema permite verificar que las tendencias descritas se sostienen aplicando distintas hipótesis sobre el grado de economías de escala en el consumo dentro de los hogares. Como es usual, los ingresos per cápita generan un límite superior para las medidas de desigualdad, mientras que si se permite cierta proporción de "bienes públicos locales" en los hogares, se eleva el ingreso de los hogares muy grandes (mayormente pobres) y disminuye la desigualdad. En el caso de las medidas de pobreza, se ajustaron las líneas de pobreza de la manera siguiente: $z^{*}=z[\mu(n)]^{1-\theta}$, donde $\mu(n)$ es el tamaño medio del hogar en la distribución (véase Deaton y Paxson, 1997).

El cuadro 2 también confirma que la evolución de la desigualdad en el período se caracteriza por un descenso entre 1976 y 1981, con un nuevo deterioro en los dos subperíodos restantes. Esta tendencia no se altera con la elección de distintas escalas de equivalencia, representadas aquí por dos distintos valores de $\theta$, aunque la desigualdad es siempre menor cuando se toman en cuenta las economías de escala dentro de los hogares. También resiste a la elección de distintas medidas de desigualdad, por lo menos en lo que toca al aumento de la desigualdad ya sea entre 1981 y 1996 o entre 1985 y 1996, como indican los resultados de dominancia de Lorenz identificados en el cuadro 3.

Los resultados para la pobreza son más ambiguos. Con respecto a la línea superior de pobreza, la inciden-

\footnotetext{
${ }^{8}$ A los tipos de cambio del mercado en 1996, equivalían aproximadamente a uno y dos dólares al día. En términos reales estas cifras serían un poco más bajas que las líneas convencionales de pobreza de uno y dos dólares diarios a precios de 1985 - que a menudo emplea el Banco Mundial para las comparaciones internacionalespor efecto de la inflación habida en los Estados Unidos en esos diez años.
} 
CUADRO 2

Brasil: Estadísticas básicas de distribución del ingreso para distintos grados de economías de escala en el consumo de los hogares

\begin{tabular}{|c|c|c|c|c|}
\hline & 1976 & 1981 & 1985 & 1996 \\
\hline Mediana (reales de 1996) ${ }^{\mathrm{a}}$ & 127.98 & 124.04 & 120.83 & 132.94 \\
\hline \multicolumn{5}{|l|}{ Desigualdad } \\
\hline Gini $-\theta=1.0$ & 0.595 & 0.561 & 0.576 & 0.591 \\
\hline Gini $-\theta=0.5$ & 0.566 & 0.529 & 0.548 & 0.567 \\
\hline $\mathrm{E}(0)-\theta=1.0$ & 0.648 & 0.542 & 0.588 & 0.586 \\
\hline $\mathrm{E}(0)-\theta=0.5$ & 0.569 & 0.472 & 0.524 & 0.534 \\
\hline $\mathrm{E}(1)-\theta=1.0$ & 0.760 & 0.610 & 0.657 & 0.694 \\
\hline $\mathrm{E}(1)-\theta=0.5$ & 0.687 & 0.527 & 0.580 & 0.622 \\
\hline $\mathrm{E}(2)-\theta=1.0$ & 2.657 & 1.191 & 1.435 & 1.523 \\
\hline $\mathrm{E}(2)-\theta=0.5$ & 2.254 & 0.918 & 1.134 & 1.242 \\
\hline \multicolumn{5}{|l|}{ Pobreza - 30 reales al mes } \\
\hline $\mathrm{P}(0)-\theta=1.0$ & 0.0681 & 0.0727 & 0.0758 & 0.0922 \\
\hline $\mathrm{P}(0)-\theta=0.5$ & 0.0713 & 0.0707 & 0.0721 & 0.0847 \\
\hline $\mathrm{P}(1)-\theta=1.0$ & 0.0211 & 0.0337 & 0.0326 & 0.0520 \\
\hline $\mathrm{P}(1)-\theta=0.5$ & 0.0235 & 0.0315 & 0.0303 & 0.0442 \\
\hline $\mathrm{P}(2)-\theta=1.0$ & 0.0105 & 0.0246 & 0.0224 & 0.0434 \\
\hline $\mathrm{P}(2)-\theta=0.5$ & 0.0132 & 0.0226 & 0.0204 & 0.0357 \\
\hline \multicolumn{5}{|l|}{ Pobreza - 60 reales al mes } \\
\hline $\mathrm{P}(0)-\theta=1.0$ & 0.2209 & 0.2149 & 0.2274 & 0.2176 \\
\hline $\mathrm{P}(0)-\theta=0.5$ & 0.2407 & 0.2229 & 0.2382 & 0.2179 \\
\hline $\mathrm{P}(1)-\theta=1.0$ & 0.0830 & 0.0879 & 0.0920 & 0.1029 \\
\hline $\mathrm{P}(1)-\theta=0.5$ & 0.0901 & 0.0875 & 0.0927 & 0.0960 \\
\hline $\mathrm{P}(2)-\theta=1.0$ & 0.0428 & 0.0525 & 0.0534 & 0.0703 \\
\hline $\mathrm{P}(2)-\theta=0.5$ & 0.0471 & 0.0508 & 0.0521 & 0.0625 \\
\hline
\end{tabular}

a Para zonas urbanas solamente y deflacionadas espacialmente.

CUADRO 3

Brasil: Resultados de dominancia estocástica

\begin{tabular}{|c|c|c|c|c|}
\hline & 1976 & 1981 & 1985 & 1996 \\
\hline 1976 & & & F & \\
\hline 1981 & & & & $\mathrm{~L}$ \\
\hline 1985 & & & & L \\
\hline 1996 & & & & \\
\hline
\end{tabular}

cia no varía en todo el período (y hasta muestra un ligero descenso para la distribución igualada). $P(l)$ y $P(2)$, sin embargo, registran incrementos en el período, que se vuelven más pronunciados y sostenidos con respecto a $\theta$ a medida que se acentúa la concavidad de la medida de pobreza. Esto sugiere que aumentaban la profundidad y la severidad de la pobreza, afectadas principalmente por la caída de los ingresos en la parte más baja de la distribución.

Estos resultados se recogen en el cuadro 3, en que una letra $L$ (o $F$ ) en la celda $(i, j)$ indica que la distribución del año $i$ presenta una dominancia estocástica de segundo orden o dominancia Lorenz (o una dominan- cia de primer orden) sobre la del año $j$. Tanto los años 1981 como 1985 muestran dominancia Lorenz sobre 1996, como se sugirió anteriormente. Hay un solo caso de dominancia de bienestar de primer orden en todo el período: el bienestar social medido en términos monetarios fue en forma no ambigua más alto en 1976 que en 1985. Todas las medidas de pobreza para ambas líneas (y para $\theta=1.0$ ) eran más altas en 1985 que en $1976 .{ }^{9}$ Es evidente que no ocurre así cuando se compara 1976 con 1996. Mientras que las medidas de pobreza muy sensibles a los niveles extremos de pobreza eran más altas en 1996, la incidencia de la pobreza para las líneas superiores (de 60 reales al mes) decae entre 1976 y 1996, lo que sugiere un cruzamiento de las funciones de distribución. El gráfico 1 muestra este cruce al dibujar los "desfiles de Pen" _ $F 1(y)$ truncados al percentil 60, para los cuatro años analizados. Nótese que mientras 1976 se sitúa en todos los

\footnotetext{
${ }^{9}$ Nótese que la dominancia de bienestar de primer orden no se sostiene ante el cambio de $\theta$ a 0.5 .
} 
GRAFICO 1

Brasil: Trayectorias Pen truncas, 1976-1996

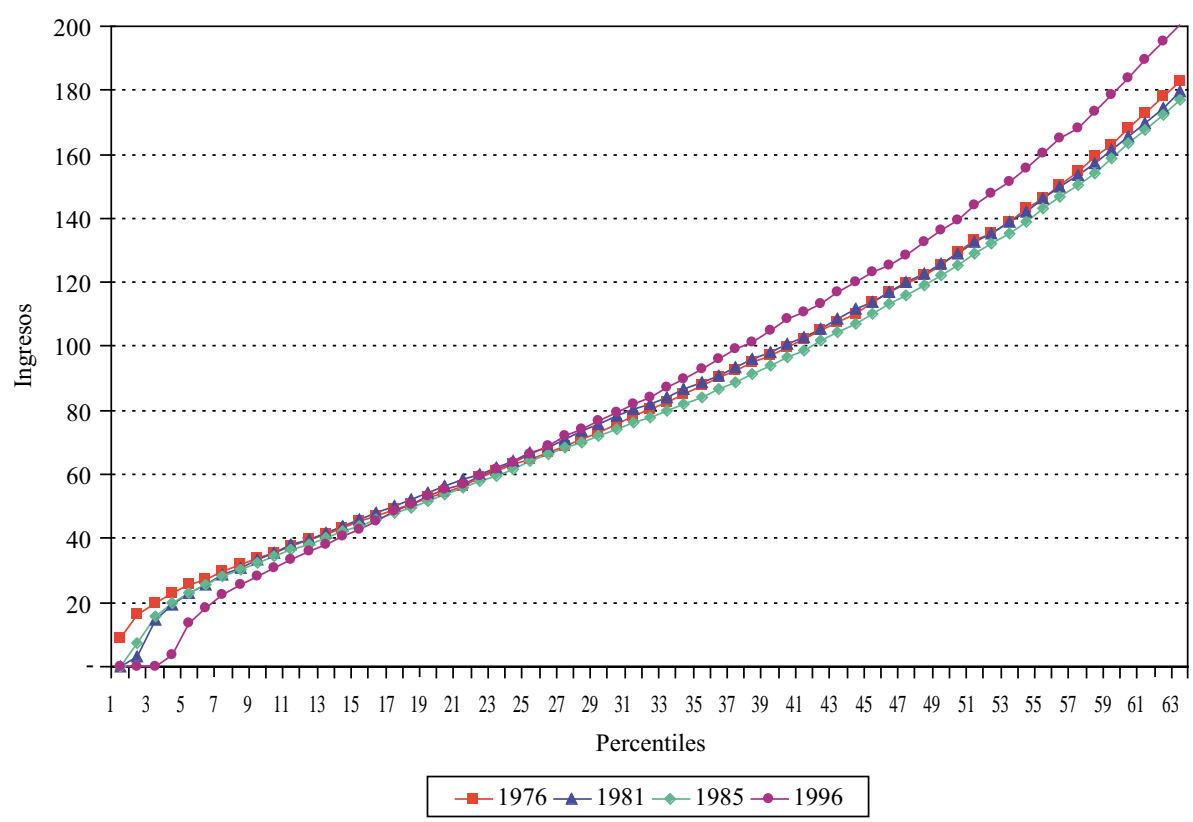

casos sobre 1985, todos los demás pares se cruzan. En particular 1976 y 1996 se cruzan a la altura del percentil 17.

Antes de analizar el modelo empleado para descomponer los cambios registrados en la distribución de los ingresos de los hogares, lo que aclarará todos esos cambios, será útil reunir alguna evidencia sobre la evolución de la educación, medida por los años de enseñanza efectivamente cursados, y sobre la participación en la fuerza de trabajo, para distintas catego- rías de la población brasileña, clasificadas por género y etnia. El cuadro 4 presenta esas estadísticas.

Como puede verse, hubo algún progreso en la educación promedio en el Brasil urbano durante este período. Los años de asistencia real a la escuela para todos los individuos de 10 y más años de edad, según aparecen en el cuadro 1, subieron de 3.2 a 5.3. En realidad este auspicioso progreso resultará de vital importancia para prevenir un aumento aún más pronunciado de la pobreza. El cuadro 4 muestra que se ha elimina-

CUADRO 4

Brasil: Estadísticas de educación y participación en la fuerza de trabajo, por género y raza ${ }^{a}$

\begin{tabular}{lcccc}
\hline & 1976 & 1981 & 1985 & 1996 \\
\hline Educación hombres (promedio años) & 3.32 & 4.04 & 4.36 & 5.20 \\
Educación mujeres (promedio años) & 3.14 & 3.99 & 4.37 & 5.43 \\
Educación raza negra y mixta (promedio años) & - & - & - & 4.20 \\
Educación raza blanca (promedio años) & - & - & - & 6.16 \\
Educación asiáticos (promedio años) & - & $74.63 \%$ & $76.04 \%$ & $71.31 \%$ \\
Participación en la fuerza de trabajo: hombres & $28.62 \%$ & $32.87 \%$ & - & $42.00 \%$ \\
Participación en la fuerza de trabajo: mujeres & - & - & - & $55.92 \%$ \\
Participación en la fuerza de trabajo: raza negra y mixta & - & - & - & $56.41 \%$ \\
Participación en la fuerza de trabajo: raza blanca & - & - & $54.88 \%$ \\
Participación en la fuerza de trabajo: asiáticos & & & & \\
\hline
\end{tabular}

a Promedio de años de educación "efectivos" para las personas de 10 o más años de edad, en zonas urbanas. Participación en fuerza de trabajo en zonas urbanas solamente. 
do la diferencia de educación entre el grupo masculino y el femenino: las mujeres mayores de 10 años tienen en promedio un poco más educación que los hombres. Evidentemente, este hecho demuestra una gran disparidad a favor de las niñas en las cohortes más recientes. Aunque un análisis por cohortes de las tendencias educacionales no entra en el ámbito del presente estudio, ${ }^{10}$ un vuelco tan acelerado pudiera estar justificando un cambio en la política gubernamental a favor de programas orientados a mantener a los varones en la escuela, sin por ello desalentar, en modo alguno, el crecimiento de la educación femenina. Por último, obsérvense las notables diferencias de educa- ción al comparar distintos grupos étnicos, en que los asiáticos se encaraman muy por encima de la media y los de raza negra y mixta quedan por debajo.

En cuanto a la participación en la fuerza de trabajo, el incremento grande y persistente de la participación femenina — de $29 \%$ a $42 \%$ entre los dos decenios- fue en parte anulado por el descenso en las tasas de participación masculina. Pese a estas tendencias, la diferencia entre la participación de hombres y mujeres sigue siendo notable, alrededor de 30 puntos porcentuales. No se manifiesta una gran diferencia de participación en la fuerza de trabajo entre los grupos étnicos.

\section{III}

\section{El modelo y la metodología de descomposición}

Analicemos nuevamente la versión brasileña del modelo general semirreducido de ingreso de los hogares y fuerza de trabajo elaborado por Bourguignon, Ferreira y Lustig (1998). El método se empleó aquí para investigar la evolución de la distribución de los ingresos per cápita de los hogares en los veinte años que corren desde mediados del decenio de 1970 hasta mediados del de 1990. Específicamente analizamos la distribución en 1976, 1981, 1985 y 1996 y simulamos los cambios entre esos años. Como se señaló anteriormente, el estudio sólo abarca las zonas urbanas del Brasil (que albergan a alrededor de tres cuartas partes de la población). El modelo general, por lo tanto, se reduce a dos categorías ocupacionales; los asalariados y los trabajadores por cuenta propia en las zonas urbanas. ${ }^{11}$ mula:

El ingreso total de los hogares se da por la fór-

$$
Y_{h}=\sum_{i=1}^{n} w_{i} L_{i}^{w}+\sum_{i=1}^{n} \pi_{i} L_{i}^{s e}+Y_{0 h}
$$

en que $w_{i}$ son los salarios totales percibidos por el individuo $i, L^{w}$ es una variable ficticia que asume el

10 Véase Duryea y Szekely (1998), que efectúan tal análisis de cohortes para Brasil y otros países latinoamericanos.

${ }^{11}$ En Brasil, los asalariados incluyen los trabajadores con o sin documentación formal (com o sem carteira). Los autoempleados son trabajadores por cuenta propia. valor de uno si el individuo $i$ es un asalariado (y de cero en caso contrario); $\pi_{i}$ representa las ganancias del trabajo por cuenta propia del individuo $i ; L^{s e}$ es una variable ficticia que asume el valor uno si el individuo $i$ es trabajador por cuenta propia (y cero en caso contrario); y $Y_{0}$ es el ingreso de otras fuentes, como ingresos de capital o por transferencia. La ecuación [1] no fue estimada econométricamente. Agrega la información del primer término de la derecha de las ecuaciones [2] y [4], del segundo de las ecuaciones [3] y [4]) y del tercero directamente de la base de datos sobre los hogares.

La ecuación de ingresos salariales se determina por;

$$
\log _{i}=X_{i}^{P} \beta^{w}+\varepsilon_{i}^{w}
$$

en que $X_{i}=\left(e d, e d^{2}, \exp , \exp ^{2}, D_{g}\right) \cdot E d$ significa años completos de educación efectiva. La experiencia (exp) se define simplemente como: edad —educación- 6 , ya que una definición más deseable debería incluir la edad a la que la persona ingresó al trabajo por primera vez, variable que no consta para $1976 .{ }^{12} D_{g}$ es una

\footnotetext{
12 Como la educación se define por el último curso completado, y por lo tanto no cuentan las repeticiones, esta definición sobreestimará la experiencia de quienes repitieron cursos en la escuela y por lo tanto rebajará el coeficiente de experiencia. Las cifras del caso no son tan altas como para influir sobre las conclusiones o tendencias.
} 
variable ficticia para el género, que asume el valor 1 para las mujeres (y cero para los hombres), $w_{i}$ son los ingresos mensuales del individuo $i, \varepsilon_{i}$ es un término residual que capta cualquier otro determinante de los ingresos, incluso otras características individuales no observadas, como el talento innato. Esta especificación muy simple se eligió para facilitar la etapa de la simulación, según se describe a continuación. De la misma manera, los ingresos de los trabajadores por cuenta propia se calculan por la ecuación siguiente;

$$
\log \pi_{i}=X_{i}^{P} \beta^{s e}+\varepsilon_{i}^{s e}
$$

Las ecuaciones [2] y [3] se calculan mediante simples mínimos cuadrados ordinarios (MCO). La ecuación [2] se calcula para todos los empleados sean o no jefes de hogar y sean o no trabajadores documentados (com o sem carteira). La ecuación [3] se estima para todos los individuos que trabajan por cuenta propia (sean o no jefes de hogar). Como los errores $\varepsilon$ probablemente no serán independientes de las variables exógenas, podría aplicarse un procedimiento de corrección de sesgo en la selección de la muestra. Sin embargo, el procedimiento estándar Heckman que se emplea para estas correcciones exige también fuertes hipótesis sobre la ortogonalidad entre los términos de error $\varepsilon$ y $\xi$ (del logit multinomial de preferencia ocupacional indicado a continuación). Las hipótesis requeridas para validar la estimación por MCO de [2] y [3] no son más complejas que las requeridas para validar los resultados de un procedimiento Heckman. Suponemos por lo tanto que todos los errores se distribuyen independientemente, y no introducimos correcciones por sesgo en la selección de la muestra en las regresiones de ingresos.

Volvamos ahora al modelo de participación de la fuerza de trabajo. Porque tenemos un mercado de trabajo de dos sectores (segmentado en los sectores de empleo asalariado y de trabajo por cuenta propia), la participación en la fuerza de trabajo y la elección del sector (opción ocupacional) deben tratarse de dos maneras diferentes. Podría suponerse que las elecciones son secuenciales, siendo la decisión de participación independiente de la opción ocupacional y ésta última condicionada por la primera. Este enfoque, que sería compatible con una estimación secuencial probit, se consideró menos satisfactorio que aquel en que los individuos hacen frente a una elección única entre tres opciones, es decir, quedar fuera de la fuerza de trabajo, trabajar como asalariado o ingresar al trabajo por cuenta propia. Tal opción puede representarse con un modelo logit multinomial. Según esa especificación, la probabilidad de estar en el estado $s(=0, w, s e)$ está dada por:

$$
P_{i}^{s}=\frac{e^{Z_{i} \gamma_{s}}}{e^{Z_{i} \gamma_{s}}+\sum_{j \neq s} e^{Z_{i} \gamma_{j}}}, \text { en que } s, j=(0, w, s e)
$$

donde las variables explicativas para los jefes de hogar y otros miembros del hogar difieren, por hipótesis, en la forma siguiente.

Para los jefes de hogar:

$Z_{1}^{h}=\left(\begin{array}{l}\frac{X_{1}^{P} ; n_{0-13}, n_{14-65}, n_{365}, \frac{1}{n_{14-65}} \sum_{-1} D_{14-65} e d,\left[\frac{1}{n_{14-65}} \sum_{-1} D_{14-65} e d\right]^{2}}{\frac{1}{n_{14-65}} \sum_{-1} D_{14-65} a g e,\left[\frac{1}{n_{14-65}} \sum_{-1} D_{14-65} a g e\right]^{2}, \frac{1}{n_{14-65}} \sum_{-1} D_{14-65} G d, D}\end{array}\right)$

Adviértase que éste es, en esencia, un modelo reducido de la oferta de trabajo, en que los ingresos propios son remplazados por las variables que los determinan, según [2] y [3].

Para los demás miembros del hogar:

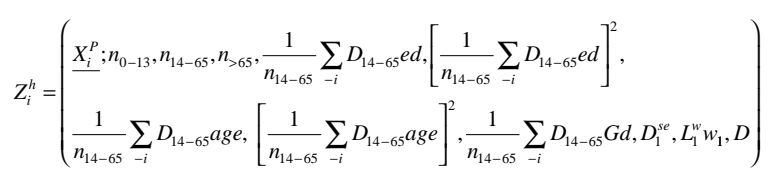

En que $n_{k-m}$ es el número de personas en el hogar cuya edad cae entre $k$ y $m ; D_{14-65}$ es una expresión ficticia que toma el valor uno para los individuos que tienen entre 14 y 65 años, $D^{s e}$ es la expresión ficticia de un jefe de hogar que trabaja por cuenta propia, y el término penúltimo representa los ingresos de un jefe de hogar asalariado. Estas dos últimas variables establecen un conducto directo para apreciar el efecto de la elección ocupacional del jefe del hogar (y posiblemente su ingreso) sobre las decisiones de participación de los demás miembros. $D$ es una variable ficticia que asume el valor de uno cuando no hay individuos de 14 a 65 años de edad en el hogar. Las sumas definidas sobre $\{-j\}$ son sumas sobre $\{\forall i \in h / j\}$.

El modelo logit multinomial de [4] corresponde al siguiente proceso de selección discreto:

$$
s=\underset{j}{\operatorname{Argmax}}\left\{U_{j}=Z_{i}^{h} \gamma_{j}+\xi_{j}, j=(0, w, s e)\right\}
$$

en que $Z$ es dada separadamente para los jefes de hogar y otros miembros del grupo; las expresiones $\xi_{j}$ son variables aleatorias con una función de densidad exponencial doble y $U_{j}$ puede interpretarse como la utili- 
dad de la alternativa $j$. Una vez que el vector $\gamma_{j}$ queda estimado por [4], y se elige un término aleatorio $\xi$, cada individuo escoge una ocupación $j$ de manera de maximizar la función de utilidad anterior.

Una vez que las ecuaciones [2], [3] y [4] han sido calculadas, habrá dos vectores de parámetros para cada uno de los cuatro años de nuestra muestra $(t \in\{1976$, 1981, 1985, 1996\}): $\beta_{t}$ derivado de las ecuaciones de ingresos tanto para asalariados como trabajadores por cuenta propia (incluyendo términos constantes $\alpha_{\mathrm{t}}$ ), $y$ $\gamma_{t}$ derivado de la ecuación de participación. Además, de la ecuación 1 tenemos $Y_{\text {oht }}$ y $Y_{h t}$. Si $X_{h t}:=\left\{X_{i}^{P}, Z_{i}^{h}\right.$ $/ \forall i \in h\}$ y $\Omega_{h t}:=\left\{\varepsilon^{w}{ }_{i}, \varepsilon^{s e}{ }_{i}, \xi_{i}^{j} / i \in h\right\}$. Podemos entonces escribir el ingreso total del hogar $h$ en el año $t$ de la forma siguiente:

$$
Y_{h t}=H\left(X_{h t}, Y_{o h t}, \Omega_{h t} ; \beta_{t}, \gamma_{t}\right) \quad h=1, \ldots, m
$$

Sobre la base de esta representación, la distribución de los ingresos del hogar:

$$
D_{t}=\left\{Y_{1 t}, Y_{2 t}, \ldots, Y_{m t}\right\}
$$

puede reformularse así:

$$
D_{t}=D\left[\left\{X_{h t}, Y_{o h t}, \Omega_{h t}\right\}, \beta_{t}, \gamma_{t}\right]
$$

en que $\{$.$\} se refiere a la distribución conjunta de las$ variables correspondientes en la población total.

Nos interesa comprender la evolución de $D_{t}$ en el tiempo, o posiblemente la de una serie de medidas alternativas sintéticas de la pobreza o la desigualdad definidas a partir de ese concepto. Sobre la base de una representación de la distribución derivada de [8], pueden interpretarse los cambios de la distribución de los ingresos en función de los efectos precio $(\beta)$, efectos de selección ocupacional $(\gamma)$, efectos de dotación $(X$. $Y_{0}$ ) y efectos residuales $(\Omega)$, según se señala en Ferreira y Paes de Barros (1999). La descomposición más sencilla se aplica a los argumentos exógenos a los hogares: es decir, $\beta s$, $\gamma s$, y la varianza de los diversos términos residuales. Cambiar los montos de $\beta$ s equivale a cambiar la rentabilidad de la variable capital humano en las ecuaciones [2] y [3]. Esto es lo que denominamos "efecto precio".

Calcular los efectos de precio y elección de ocupación es tarea bastante sencilla, una vez que se han estimado los parámetros exógenos correspondientes. Para calcular los efectos sobre la dotación individual se requiere otra operación más, ya que los elementos de los vectores $X$ e $Y$ están distribuidos conjuntamente, $y$ un cambio en el valor de cualquier variable debe considerarse como condicional de todas las otras características observables.

Específicamente, si nos interesa el efecto de un cambio en la distribución de una sola variable concreta $X_{k}$ sobre la distribución de los ingresos de los hogares entre los tiempos $t$ y $t^{\prime}$, es primero necesario identificar la distribución de $X_{k}$ que depende de otras características atinentes $X_{-k}$ (y posiblemente de otros ingresos $Y_{0}$ ). Esto puede lograrse con la regresión de $X_{k}$ sobre $X_{-k}$ en las fechas $t$ y $t^{\prime}$ de la forma siguiente;

$$
X_{k i t}=X_{-k i t} \mu_{t}+u_{k i t}
$$

en que $k$ es la variable, $i$ es el individuo y $t$ es la fecha. El vector de residuos $u_{k i t}$ representa los efectos en $X_{k}$ de las características no observables (que se supone son ortogonales a $X_{-k}$ ). El vector $\mu_{\mathrm{t}}$ es un vector de coeficientes que capta la dependencia de $X_{k}$ de las verdaderas variables exógenas $X_{-k}$, en el tiempo $t$. En aras de la simplicidad, supongamos que los términos de error $u$ se distribuyen normalmente con una media de 0 y una desviación estándar de $\sigma_{t}$.

La misma ecuación, evidentemente, puede calcularse en la fechas $t$ ', lo que generará un vector correspondiente de coeficientes $\mu_{1}$ y un error estándar de los residuos dado por $\sigma_{t}$. Estaremos entonces en condiciones de simular el efecto de un cambio en la distribución condicional de $X_{k}$ de $t$ a $t^{\prime}$, reemplazando los valores observados de $X_{k i t}$ en la muestra observada en el tiempo $t$, por:

$$
X_{k i t}^{*}=X_{-k i t} \mu_{t^{\prime}}+u_{k i t} \frac{\sigma_{t^{\prime}}}{\sigma_{t}}
$$

La contribución de un cambio en la distribución de la variable $X_{k}$ al cambio en la distribución de los ingresos entre $\mathrm{t}$ y $\mathrm{t}^{\prime}$ puede ahora expresarse así:

$$
\begin{aligned}
R_{t t^{\prime}}^{x^{*}} & =D\left[\left\{X_{k i t^{*}} *, X_{-k i t}, Y_{o h t}, \Omega_{h t}\right\}, \beta_{t}, \gamma_{t}\right] \\
& -D\left[\left\{X_{k i t}, X_{-k i t}, Y_{o h t}, \Omega_{h t}\right\}, \beta_{t}, \gamma_{t}\right]
\end{aligned}
$$

En este estudio hacemos cuatro estimaciones de regresión como la de [9] y por lo tanto cuatro simulaciones como la de [10]. Las cuatro variables estimadas son $X_{k}=\left\{n_{0-13}, n_{14-65}, n_{>65}, e d\right)$. En el caso de la regresión relativa a la educación, el vector de las va- 
riables explicativas $X_{-k i t}$ fue (l, edad, edad ${ }^{2}, G_{d}$; variables ficticias regionales ${ }^{13}$ ). En el caso de las regresiones con el número de miembros del hogar entre determinados tramos de edad usado como variable dependiente, el vector $X_{-k i t}$ era (1, edad, edad ${ }^{2}$, ed, ed ${ }^{2}$, variables ficticias regionales), en que la edad y la educa- ción corresponden al jefe del hogar. La simulación posible con estas estimaciones nos permite investigar los efectos de la evolución en la distribución de los logros educacionales y la estructura demográfica sobre la distribución del ingreso. Examinemos ahora los resultados de la etapa de estimación del modelo.

\section{IV}

\section{Estimación del modelo}

Los resultados de la estimación por MCO de la ecuación [2] para los asalariados (del sector formal e informal) aparecen en el cuadro 5. Los resultados estáticos no son sorprendentes. Todos los indicadores son significativos y muestran los signos esperados. Los coeficientes de la enseñanza y sus cuadrados son positivos y significativos. El efecto de la experiencia (definida como [edad —enseñanza-6]) es positivo, pero no cóncavo. El indicador ficticio de género (mujer $=1$ ) es negativo, significativo y grande.

CUADRO 5

Brasil: Regresión de ingresos para los asalariados (ecuación [2]) ${ }^{\mathrm{a}}$

\begin{tabular}{lcccc}
\hline Años & 1976 & 1981 & 1985 & 1996 \\
\hline Intercepto & 4.350 & 4.104 & 3.877 & 4.256 \\
& $(0.0001)$ & $(0.0001)$ & $(0.0001)$ & $(0.0001)$ \\
Educación & 0.123 & 0.136 & 0.129 & 0.080 \\
& $(0.0001)$ & $(0.0001)$ & $(0.0001)$ & $(0.0001)$ \\
Educación ${ }^{2}$ & 0.225 & 0.181 & 0.283 & 0.438 \\
(x 100) & $(0.0001)$ & $(0.0001)$ & $(0.0001)$ & $(0.0001)$ \\
Experiencia & 0.075 & 0.085 & 0.087 & 0.062 \\
& $(0.0001)$ & $(0.0001)$ & $(0.0001)$ & $(0.0001)$ \\
Experiencia $^{2}$ & -0.105 & -0.119 & -0.121 & -0.080 \\
(x 100) & $(0.0001)$ & $(0.0001)$ & $(0.0001)$ & $(0.0001)$ \\
& & & & \\
Género & -0.638 & -0.590 & -0.635 & -0.493 \\
$(1=$ mujeres) & $(0.0001)$ & $(0.0001)$ & $(0.0001)$ & $(0.0001)$ \\
$\mathrm{R}^{2}$ & & & & \\
& 0.525 & 0.538 & 0.547 & 0.474 \\
\hline
\end{tabular}

Fuente: Cálculos de los autores sobre la base de la encuesta nacional de hogares PNAD (IBGE, 1976, 1981, 1985 y 1996). Las cifras entre paréntesis son valores $\mathrm{P}$.

\footnotetext{
${ }^{13}$ Las variables ficticias regionales se refieren a las cinco regiones del Brasil: Norte, Nordeste, Centro-Occidente y Sur, siendo el Sudeste la categoría por omisión.
}

La evolución dinámica es más interesante. Entre 1976 y 1996, el perfil ingreso-enseñanza cambió de forma. Después de subir a fines del decenio de 1970, el componente lineal disminuyó considerablemente entre 1981 y 1996. Entretanto, el coeficiente del cuadrado de años de educación se redujo hasta 1981 pero luego subió a más del doble hacia 1996, cerrando el período a un valor muy por encima del inicial de 1976. En general, la relación se volvió más convexa, lo que sugiere una línea más escarpada de los rendimientos marginales de la educación en los tramos superiores. Sin embargo, al dibujar la parábola que representa la relación entre ingresos parciales y la enseñanza, de la ecuación [2], domina el descenso del término lineal. El perfil asciende entre 1976 y 1981 y nuevamente en 1985, antes de sufrir una caída precipitada (y volverse más convexa) hasta 1996 (gráfico 2). El efecto neto en todo el período fue de caída en los rendimientos acumulativos de la educación (de cero a $t$ años) en toda la gama, pese al alza de los rendimientos marginales de la enseñanza en los tramos superiores. Son evidentes las repercusiones para la pobreza y la desigualdad: los efectos de precios llevan, en igualdad de las demás condiciones, a un aumento de la primera y a una declinación de la segunda.

Los rendimientos de la experiencia también aumentaron entre 1976 y 1981 y entre 1981 y 1985 , con una curva cóncava y un máximo de alrededor de 35 años de experiencia (gráfico 3). Sin embargo, entre 1985 y 1996 se registró un gran descenso en los rendimientos acumulativos de la experiencia respecto a 1976, hasta 50 años de experiencia. La relación se volvió menos cóncava y los rendimientos máximos subieron hasta alrededor de los 40 años. En todo el período el efecto precio de la experiencia fue ligeramente desigualador (aunque contribuyó a los aumentos de la desigualdad hasta 1985, luego ocurrió lo contrario) con serias repercusiones de aumento de la pobreza. 
GRAFICO 2

Brasil: Rendimientos cuadráticos de la enseñanza (Asalariados)

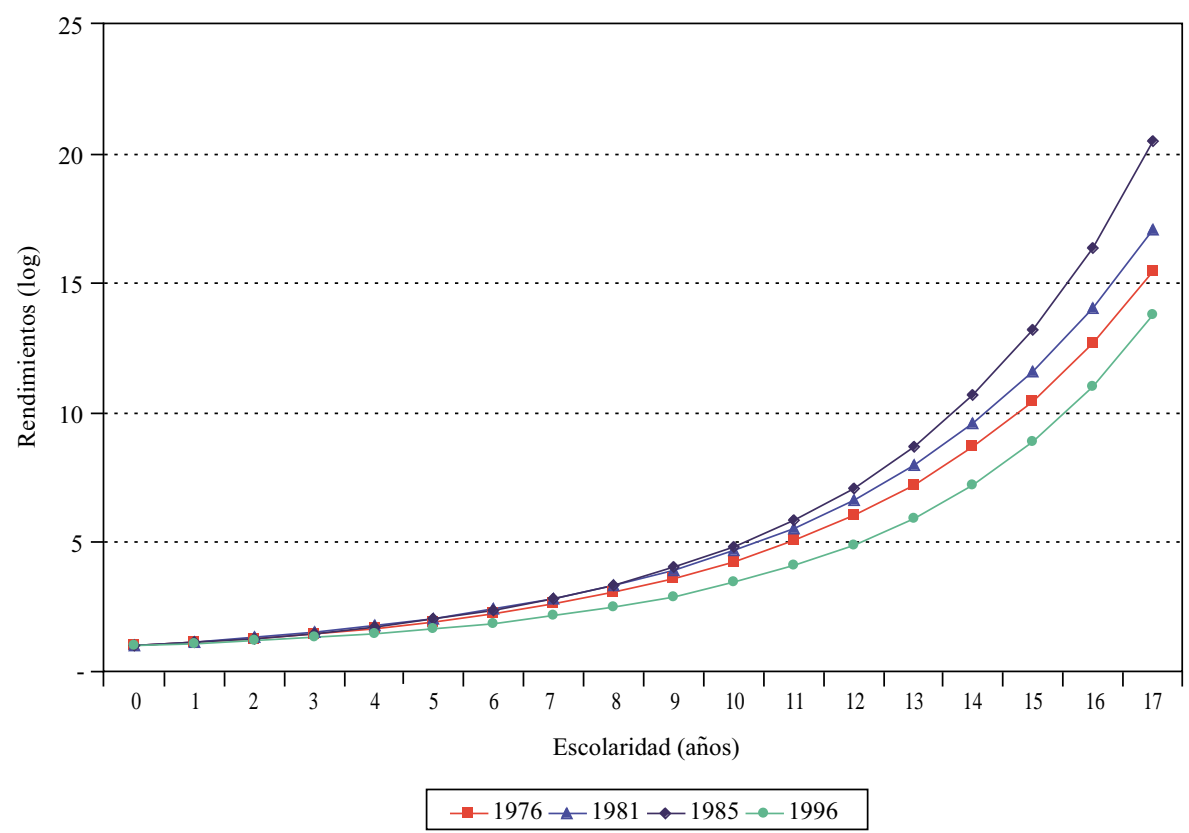

GRAFICO 3

Brasil: Rendimientos cuadráticos de la experiencia (Asalariados)

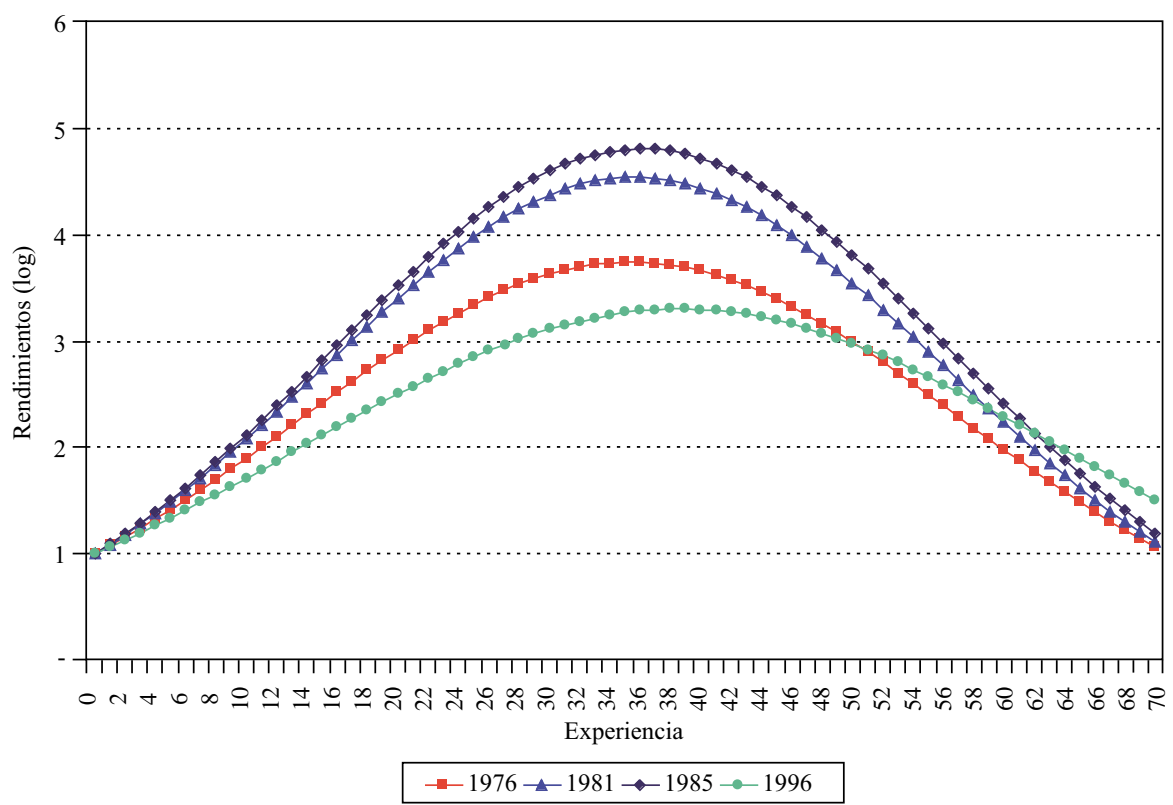


La única novedad alentadora es la menor disparidad entre los ingresos de hombres y mujeres.

Aunque los ingresos femeninos, controlados tanto por educación como por experiencia, siguieron bastante más bajos en los cuatro años estudiados, lo que sugiere que puede haber alguna discriminación en el mercado del trabajo, hubo, sin embargo, un descenso de este fenómeno entre 1976 y 1996. Este efecto, como veremos en la simulación descrita en la sección $\mathrm{V}$, tiene leves repercusiones igualadoras y de reducción de la pobreza. Volvamos ahora a la ecuación [3] que trata de explicar los ingresos de los trabajadores por cuenta propia con la misma serie de variables independientes de la ecuación [2]. Los resultados aparecen en el cuadro 6. El cuadro muestra que la educación es también una determinante importante de los ingresos para el sector de trabajadores por cuenta propia. El coeficiente en el término lineal tiene un valor más alto en todos los años que para los asalariados pero el del término cuadrático es más bajo. Esto implica que, ceteris paribus, los rendimientos de una baja educación podrían ser más altos para los trabajadores por cuenta propia que para los asalariados, pero que a la postre bajarían a medida que aumentara la educación. Ello influirá sobre la elección ocupacional, estimada en la ecuación [4]. Dinámicamente se observó la misma tendencia de los asalariados: los coeficientes del término lineal decaen con el tiempo, pero la relación se vuelve más convexa. ${ }^{14}$ Los coeficientes de experiencia y de experiencia al cuadrado siguen una evolución similar que la observada para los asalariados. Una vez más, el rendimiento acumulativo para la experiencia decayó en la generalidad de los años de 1976 a 1996, contribuyendo así al aumento observado de la pobreza. El efecto de ser mujer es aún más negativo en este sector que en el asalariado. También decayó entre 1976 y 1996, pese a un aumento temporal de la disparidad en el decenio de 1980.

Valga una palabra de advertencia antes de seguir adelante. Todos los resultados de la estimación consignados anteriormente se basan en ecuaciones en que los ingresos totales son variables independientes. Los cambios de coeficientes, por lo tanto, se reflejarán no sólo en el rendimiento horario de determinada característica, sino también en cualquier respuesta por el lado de la oferta que pudiera haber ocurrido. El análisis debe interpretarse a la luz de estas consideraciones.

${ }^{14}$ En este caso, se transformó de cóncava en convexa.
CUADRO 6

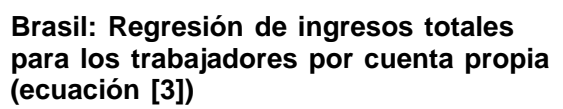

\begin{tabular}{lcccc}
\hline Años & 1976 & 1981 & 1985 & 1996 \\
\hline Intercepto & 4.319 & 4.192 & 3.853 & 4.250 \\
& $(0.0001)$ & $(0.0001)$ & $(0.0001)$ & $(0.0001)$ \\
Educación & 0.196 & 0.148 & 0.165 & 0.114 \\
& $(0.0001)$ & $(0.0001)$ & $(0.0001)$ & $(0.0001)$ \\
Educación ${ }^{2}$ & -0.206 & 0.021 & 0.012 & 0.219 \\
(x 100) & $(0.0001)$ & $(0.4892)$ & $(0.6545)$ & $(0.0001)$ \\
Experiencia & 0.074 & 0.079 & 0.084 & 0.063 \\
Experiencia $^{2}$ & $(0.0001)$ & $(0.0001)$ & $(0.0001)$ & $(0.0001)$ \\
(x 100) & -0.101 & -0.108 & -0.111 & -0.082 \\
Género & $(0.0001)$ & $(0.0001)$ & $(0.0001)$ & $(0.0001)$ \\
& -1.092 & -1.148 & -1.131 & -0.714 \\
$\mathrm{R}^{2}$ & $(0.0001)$ & $(0.0001)$ & $(0.0001)$ & $(0.0001)$ \\
\hline
\end{tabular}

Fuente: Cálculos de los autores sobre la base de la encuesta nacional de hogares PNAD (IBGE, 1976, 1981, 1985 y 1996).

a Las cifras entre paréntesis son valores $\mathrm{P}$.

Volvamos ahora a la estimación del logit multinomial en la ecuación [4]. Este se calculó separadamente para el jefe del hogar y los demás, ya que la serie de variables explicativas era ligeramente distinta en uno y otro caso (véase la descripción de los vectores $Z_{1}$ y $Z_{i}$ en la sección III). ${ }^{15}$

Para los jefes de hogar, no hubo una relación significativa, en ningún momento, entre la educación y la probabilidad de elegir el trabajo asalariado o decidir abandonar la fuerza de trabajo. El efecto dominante sobre las opciones ocupacionales de los jefes de hogares urbanos en este período fue el descenso marcado en el término constante que afecta la probabilidad de ingreso en uno $u$ otro sector productivo, en contraposición a quedar fuera de la fuerza de trabajo, o en el desempleo. Como es captado por la constante, este efecto no se relaciona ni con la educación ni con la experiencia del jefe del hogar, ni con la dotación de su hogar. Lo interpretamos, en cambio, como el efecto del mercado del trabajo, que lleva a una menor participación del trabajo asalariado. ${ }^{16} \mathrm{Se}$ demostrará en la sección siguiente, que trata de las simulaciones de opción

\footnotetext{
15 Por limitaciones de espacio no podemos presentar los cuadros que contienen estas estimaciones. Se encuentran en Ferreira y Paes de Barros (1999, apéndice 3).

${ }^{16}$ En el marco de la "opción ocupacional", éstos representan cambios en las limitaciones que afectan a esas opciones.
} 
ocupacional, que este efecto es a la vez desigualador y empobrecedor.

Para los demás miembros del hogar, la escolaridad no parece aumentar la probabilidad de elegir el trabajo asalariado frente a la opción de quedar fuera de la fuerza de trabajo, ya que la relación cambia de forma cóncava a convexa (y débil) en el período. También se fortalece la probabilidad del trabajo por cuenta propia frente a la opción de estar fuera de la fuerza de trabajo en ambos períodos, aunque esta relación siguió siendo cóncava. El número de hijos por hogar significativamente desalentó la participación en ambos sectores, aunque en mayor medida en el sector asalariado. El cambio en los términos constantes fue mucho menor para los jefes de hogar, lo que sugiere que las condiciones desfavorables del mercado de trabajo perjudican en mayor grado al sostén primario del hogar. Por consiguiente, se observará que el efecto de las opciones ocupacionales para otros miembros del hogar sobre la pobreza y la desigualdad será mucho menor que para los jefes de hogar.

Los resultados de la estimación de la ecuación [9] en que la variable dependiente es la escolaridad de los individuos de 10 años y más con regresión frente al vector (1, edad, edad ${ }^{2}, G_{d}$, variables ficticias regionales) se dan también en Ferreira y Paes de Barros
(1999). Con el tiempo ocurre un aumento considerable en el valor del intercepto, que dará valores proyectados más altos para los logros educativos, controlando por edad, género y ubicación regional. Además, la expresión ficticia de género evolucionó de alta y negativa a positiva y significativa, lo que sugiere que en el Brasil las mujeres han alcanzado y superado a los hombres en su progreso educativo en los últimos veinte años. El efecto de la edad individual es estable y las disparidades regionales persisten: el sur y el sureste aventajados con respecto a las tres regiones centrales $\mathrm{y}$ el norte.

Al regresar el número de miembros del hogar entre los 0-13 años de edad, 14-65 y sobre 65 (respectivamente), sobre el vector (1, ed, ed ${ }^{2}$, edad, edad ${ }^{2}$, variables ficticias regionales) resulta que la educación del jefe del hogar tiene un efecto grande, negativo y significativo sobre la demanda de hijos, de manera que a medida que suben los niveles de enseñanza, tendería a decaer el tamaño de la familia, si no cambiaran las demás condiciones. Además, puede inferirse cierto grado de convergencia entre las regiones en cuanto al tamaño de la familia, ya que los coeficientes regionales ficticios de 1976 descienden para todas las regiones (con respecto a la región sudoriental) y decaen a menos de la mitad de su valor en 1996.

\section{$\mathrm{V}$}

\section{Los resultados de la simulación}

Luego de estimar las ecuaciones de ingresos para ambos sectores del modelo - asalariados [2] y trabajadores por cuenta propia [3]—, ecuaciones de participación para los jefes del hogar y los demás miembros [4], y ecuaciones de dotación [9] para la determinación exógena de la enseñanza y la composición del hogar, estamos en situación de efectuar las descomposiciones descritas por Bourguignon, Ferreira y Lustig (1998). Estas simulaciones, como se señaló anteriormente, se efectúan para toda la distribución. Los resultados se resumen en el cuadro 7, indicando el ingreso medio per cápita de los hogares $\mu(y)$, cuatro índices de desigualdad (el coeficiente de Gini, el índice Theil-L [E(0)], el índice Theil-T [E(1)] y $E(2)$, y los tres miembros estándares de la clase $P(\alpha)$ de medidas de pobreza Foster-Greer-Thorbecke, $\alpha=0,1,2$, computadas con respecto a dos líneas de pobreza según el ingreso mensual: la línea de indigencia de 30 reales al mes y una línea de pobreza de 60 reales al mes (expresadas ambas a precios de São Paulo de 1996). ${ }^{17}$

El cuadro 7 contiene información sobre gran número de cambios económicos simulados, siempre aplicando una combinación de coeficientes de 1996 a la población de 1976. A fin de resolver los dos enigmas planteados en la introducción - a saber, el incremen-

\footnotetext{
${ }^{17}$ Las cifras del cuadro 7 y las demás que aparecen en esta sección se refieren a la simulación de aplicar a 1976 los coeficientes estimados para 1996. Se hicieron ejercicios similares para 1981 y 1985 , los que se describen en Ferreira y Paes de Barros (1999). Asimismo, se llevó a cabo la simulación inversa de aplicar a 1996 los coeficientes para 1976, y las direcciones y en general las magnitudes de los cambios confirmaron los resultados que se presentan más adelante.
} 
Brasil: Simulación de pobreza y desigualdad para 1976, empleando coeficientes de 1996

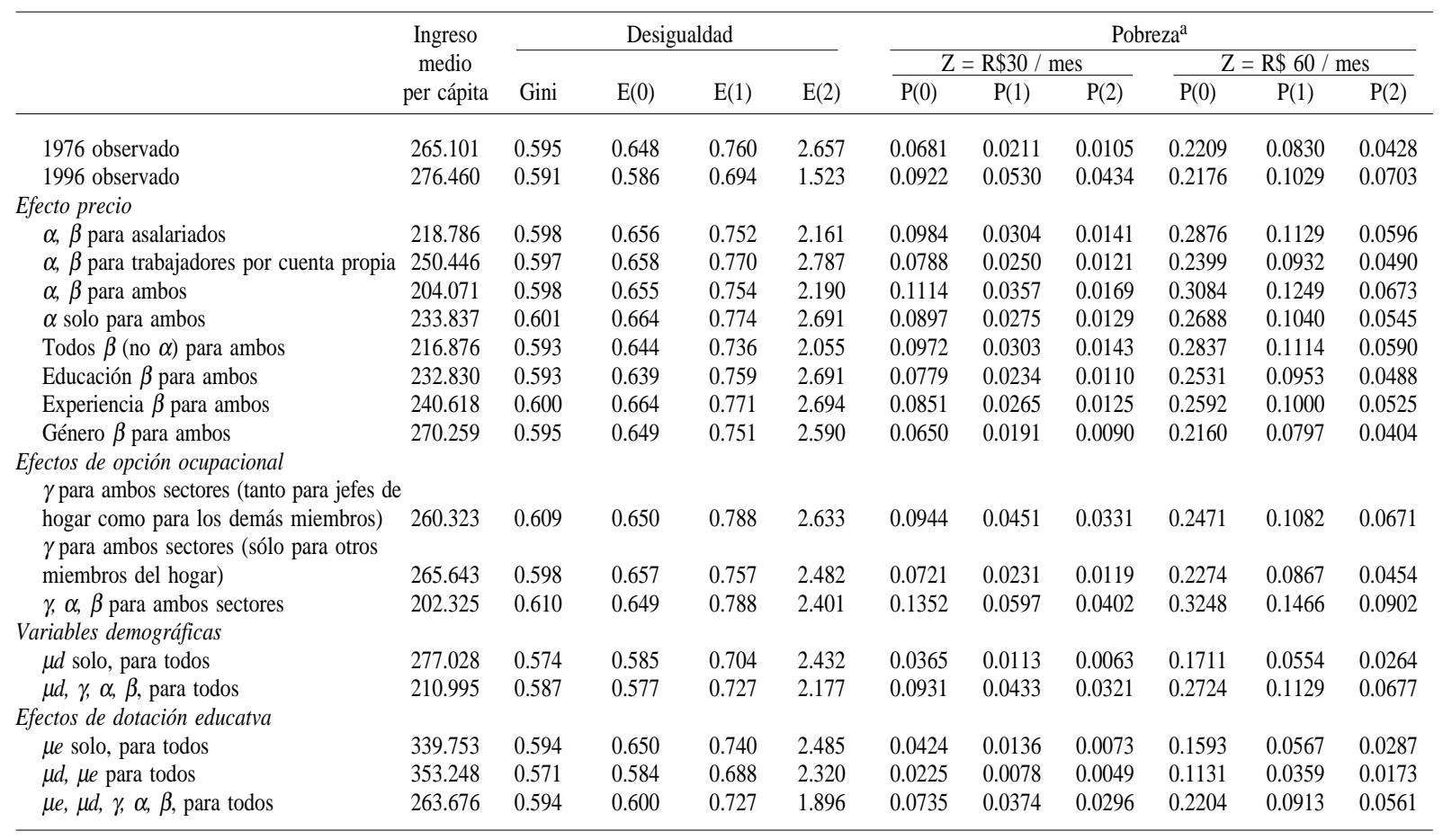

Fuente: Basado en la encuesta de hogares PNAD, IBGE (1976 y 1996).

a $\mathrm{Z}=$ línea de pobreza.

to de la pobreza urbana extrema entre 1976 y 1996 pese a un crecimiento (aunque lento) que reducía la desigualdad y la coexistencia de un deterioro del mercado laboral con una pobreza estable de "línea superior"- observamos las diferencias en los logaritmos de ingresos entre la distribución simulada de los ingresos per cápita de los hogares y la observada para 1976, con relación a varias simulaciones del cuadro 7 .

El gráfico 4 ilustra el efecto combinado de precios $(\alpha$ y $\beta$ ), separadamente para los asalariados y los trabajadores por cuenta propia. Como puede apreciarse, estos efectos fueron negativos (es decir, habrían supuesto un ingreso más bajo en 1976) para todos los percentiles. Las pérdidas fueron mayores para los asalariados que para los trabajadores por cuenta propia, y para estos últimos fueron regresivas. Estas pérdidas son justamente lo que cabría esperar de un decaimiento de los perfiles parciales de ingreso-educación e ingreso-experiencia que aparecen más atrás en los gráfi$\cos 2$ y 3 .

En el gráfico 5 adoptamos un enfoque diferente para analizar los efectos de precio, al representar las diferencias de ingreso para cada simulación del efecto precio (para ambos sectores en conjunto) y entonces agregarlos. Como cabría esperar de los gráficos 2 y 3 , los rendimientos de la educación y la experiencia son ambos pauperizantes. El cambio en los rendimientos parciales de la educación por sí solo tiene un leve efecto igualador (como puede verse en el cuadro 7). El cambio en los rendimientos parciales de la experiencia es desigualador y pauperizante. El cambio en el intercepto, calculado en los valores medios de las variables independientes, es negativo siempre. Esto constituye un indicador vicario del efecto de "crecimiento puro", y capta efectos sobre los ingresos derivados de procesos que no se reflejan en la educación, la experiencia, el género, o las características no observadas de los trabajadores individuales. Tiene por objeto captar los efectos de la acumulación de capital, la innovación gerencial y técnica, las condiciones de la política macroeconómica y otros factores que probablemente determinen el crecimiento económico, que no se incluyen explícitamente en la ecuación de Mincer utilizada. Su efecto negativo sobre esta simulación 


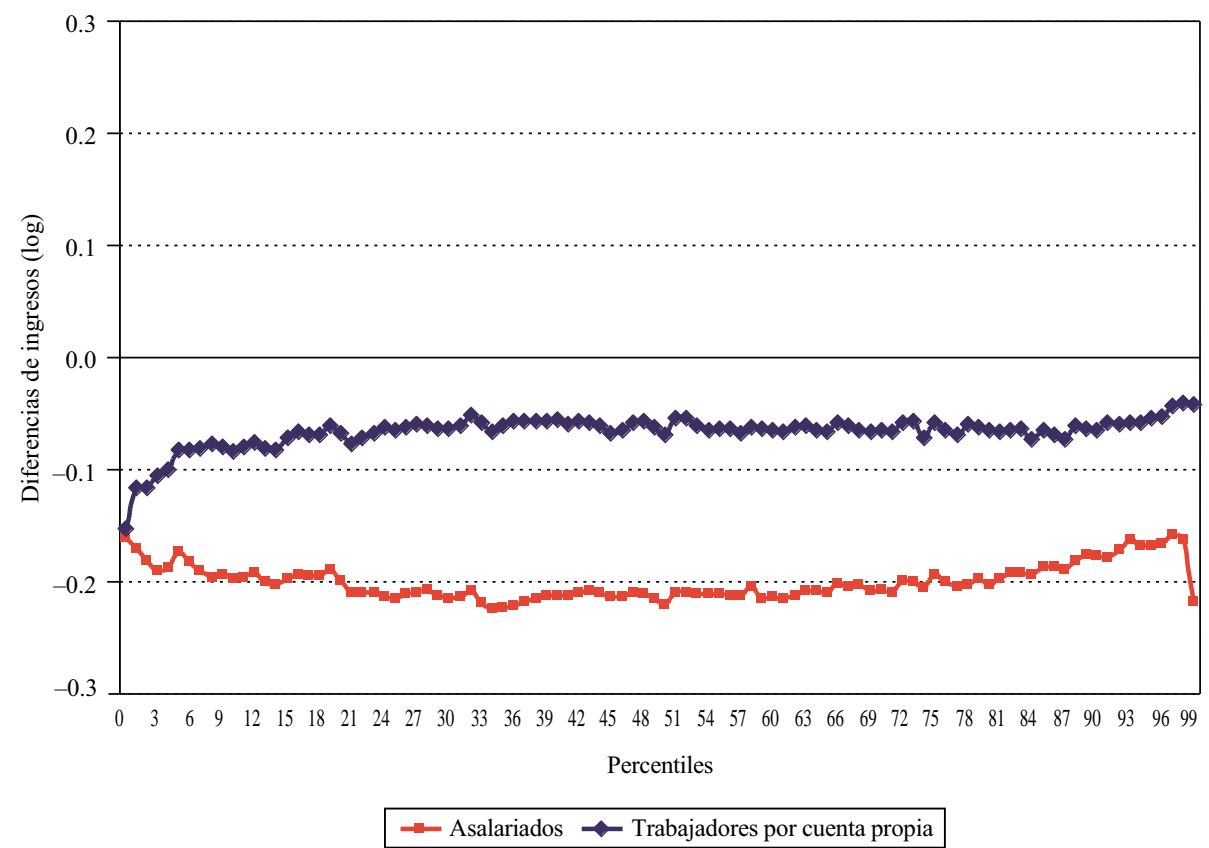

GRAFICO 5

Brasil: Efectos de precios separada y conjuntamente (ambos sectores)

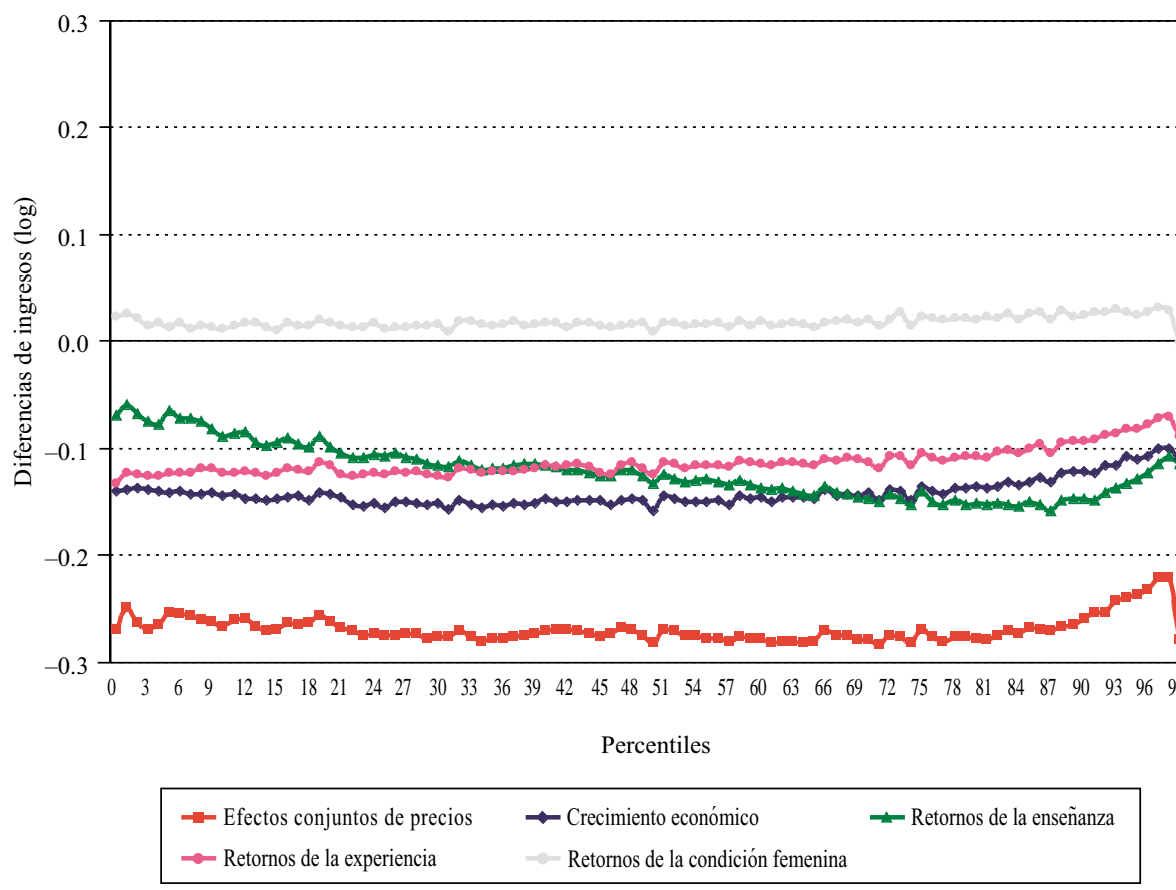

Fuente: IBGE, 1976 y 1996. 
sugiere que estos factores fueron pauperizantes en el Brasil urbano en el período examinado.

El único signo alentador, nuevamente, proviene de la simulación por géneros, que da cuenta de un efecto de reducción de la pobreza como resultado de la menor diferencia de ingresos entre hombres y mujeres que aparece en los cuadros 5 y 6 . Este efecto, sin embargo, no fue suficiente como para compensar los efectos negativos conjuntos de los demás efectos de precio. Como indica la línea gruesa en la parte inferior del gráfico 6, el efecto combinado de imponer los parámetros de opción ocupacional de 1996 de las dos ecuaciones de Mincer sobre la población de 1976 resultó considerablemente empobrecedor.

El gráfico 6 representa (el logaritmo de) las diferencias de ingreso entre la distribución obtenida al imponer los parámetros de opción ocupacional de 1996 - el vector $\gamma$ del logit multinomial de la ecuación [4] - a la población de 1976, y la distribución observada de 1976. Lo hace tanto para todos los individuos (línea inferior) como para los que no son jefes de hogar (línea superior). El efecto de este cambio simulado en la opción ocupacional y participación en la fuerza de trabajo es muy empobrecedor y desigualador, como confirman los índices respectivos del cuadro 7. Sugiere que hay un grupo de personas que voluntaria o involuntariamente abandonan la fuerza de trabajo, o ingresan al desempleo, o están relegadas a ocupaciones muy mal remuneradas, probablemente en el sector informal, y que se están pauperizando crecientemente.

Al combinar los efectos negativos de precio y opción ocupacional, se tiene una visión general de las condiciones del mercado del trabajo urbano en el Brasil en este período. Esto se presenta en el gráfico 7, en que la curva inferior representa las diferencias de ingresos per cápita de los hogares con una distribución en que todos los $\alpha s, \beta s$ y $\gamma_{s}$ cambian, y la distribución observada de 1976. Muestra cuán poderoso fue el efecto conjunto, en cuanto al aumento de la pobreza y de la desigualdad, de los cambios en los precios del mercado de trabajo y los parámetros de opción ocupacional sobre la distribución de 1976.

A estas alturas puede plantearse claramente el segundo enigma: dadas estas circunstancias del mercado del trabajo, ¿qué factores pueden explicar el que la media de los ingresos subiera, la pobreza de "línea superior" no subiera y la desigualdad pareciera haber disminuido ligeramente? La primera parte de la respuesta se da en el gráfico 8 en que la curva superior representa la diferencia de ingresos (log) de una distribución derivada de imponer en la población de 1976

GRAFICO 6

Brasil: Efectos de la opción ocupacional

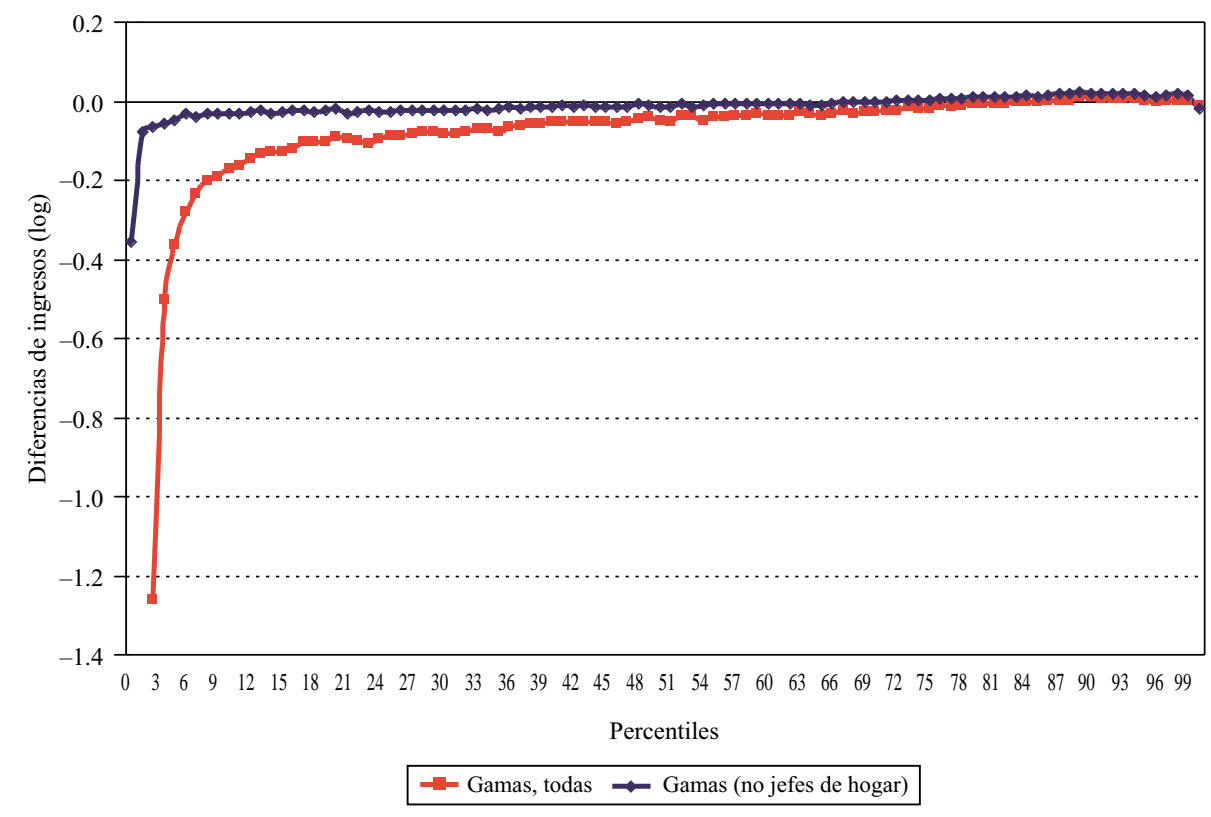

Fuente: IBGE, 1976 y 1996. 
GRAFICO 7

Brasil: El mercado de trabajo: combinación de los efectos de precio y opción ocupacional

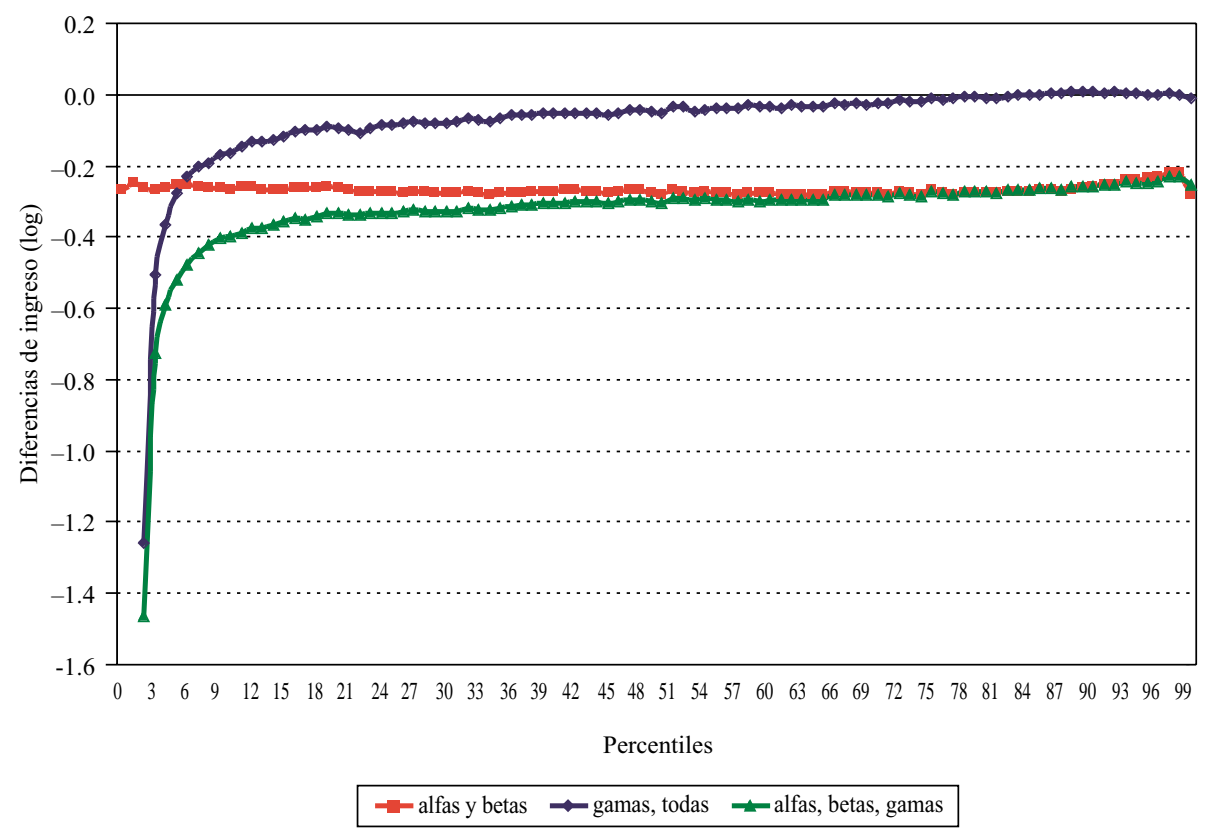

Fuente: IBGE, 1976 y 1996.

GRAFICO 8

Brasil: Efectos demográficos

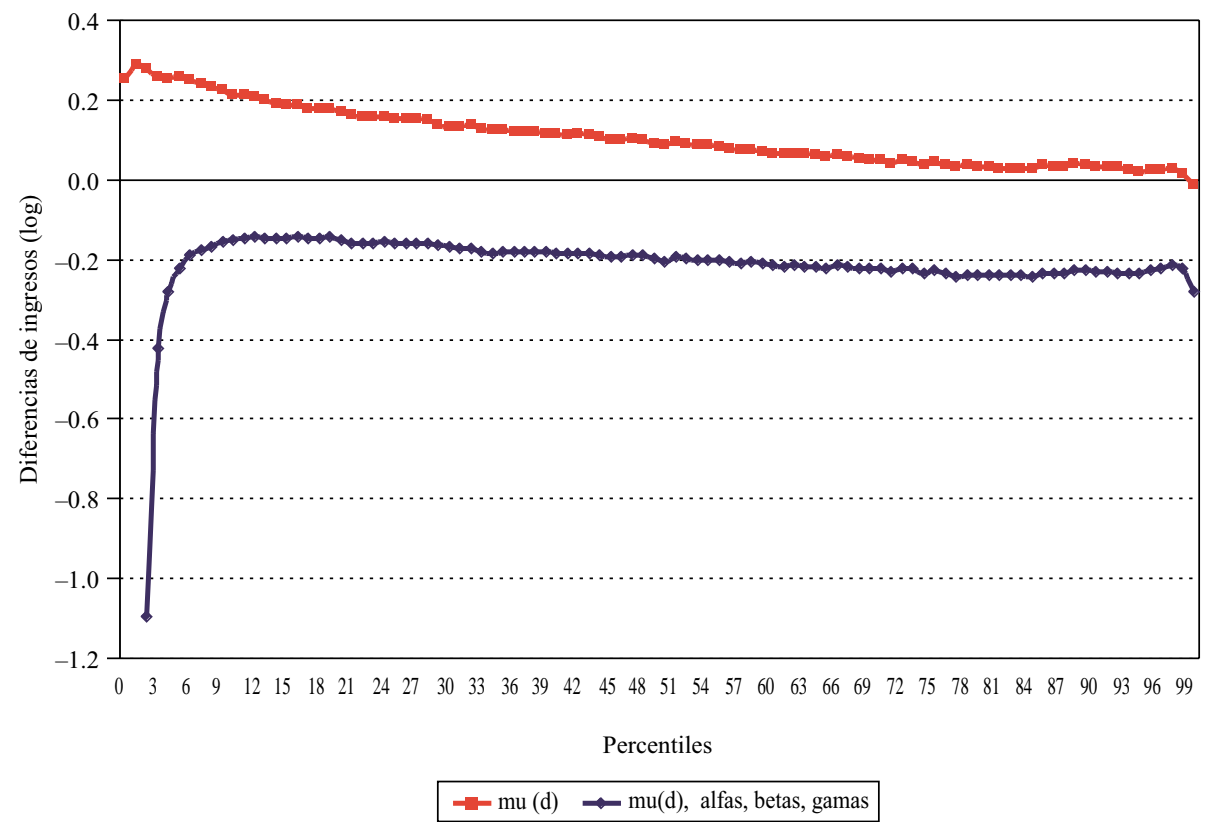

Fuente: IBGE, 1976 y 1996. 
la transformación (10) de la estructura demográfica de la población. Los cambios en los parámetros $\mu_{d}$ (y en las varianzas de los residuos en la regresión correspondiente) tienen un efecto positivo sobre los ingresos en todos los percentiles y en forma igualadora. Sin embargo, cuando se combinan en una simulación en que los valores de todos los $\alpha, \beta$ y $\gamma$ también cambian, puede apreciarse que el efecto demográfico positivo sigue siendo anulado. Sin embargo, es evidente que la reducción de la relación de dependencia y por consiguiente del tamaño de la familia en las zonas urbanas del Brasil en este período tuvo un importante efecto mitigante sobre la distribución de los ingresos.

Queda una pieza final del rompecabezas, necesaria para explicar por qué el deterioro de las condiciones del mercado del trabajo no tuvo un peor impacto sobre la pobreza. Y es que, como debía ser evidente al considerar el aumento en los años promedio de escolaridad efectiva, señalado en el cuadro 1 , hubo un desplazamiento hacia la derecha en la función de la educación. Esto se aprecia en el gráfico 9, que muestra que los logros educativos fueron más marcados en los niveles inferiores de educación y, por ende, presumiblemente entre los pobres.

El progreso en los logros educativos en toda la distribución del ingreso, pero sobre todo entre los estratos más pobres, tiene efectos tanto directos como indirectos sobre los ingresos. Los efectos directos se aprecian en las ecuaciones [2] y [3] en que los ingresos son funciones positivas de la educación. Los efectos indirectos se manifiestan tanto sobre las opciones ocupacionales de los individuos como sobre el impacto adicional que tiene la educación al reducir la demanda de hijos y, por lo tanto, el tamaño de la familia. Así, es tarea bastante compleja efectuar la simulación del efecto de la enseñanza. ${ }^{18} \mathrm{Al}$ completarla, se observa en el gráfico 10 un mejoramiento más bien plano en los ingresos (log) en toda la distribución (el efecto de escalamiento). Sin embargo, cuando se combina con cambios en los parámetros de las ecuaciones demográficas, el efecto se fortalece y se vuelve no sólo más reductor de la pobreza, sino ligeramente igualador. La línea inferior en el gráfico 10 se ajusta a este patrón y combina ambos efectos con la variación de $\alpha s, \beta s$ y $\gamma s$. El resultado es notable: esta compleja simulación combinada sugiere que todos estos efectos, durante 20 agitados años, se cancelan unos a otros casi totalmente a partir del percentil 15. De ahí la escasa variación en la pobreza de la "línea superior". Sin embargo, del

\footnotetext{
${ }^{18}$ Nótese que los efectos no se suman simplemente. El efecto de un mayor logro educativo se simula en todas las ecuaciones en que aparece en el modelo, afectando así las opciones de fecundidad y el status ocupacional, así como los ingresos.
}

Brasil: Desplazamiento en la distribución de la escolaridad, 1976-1996

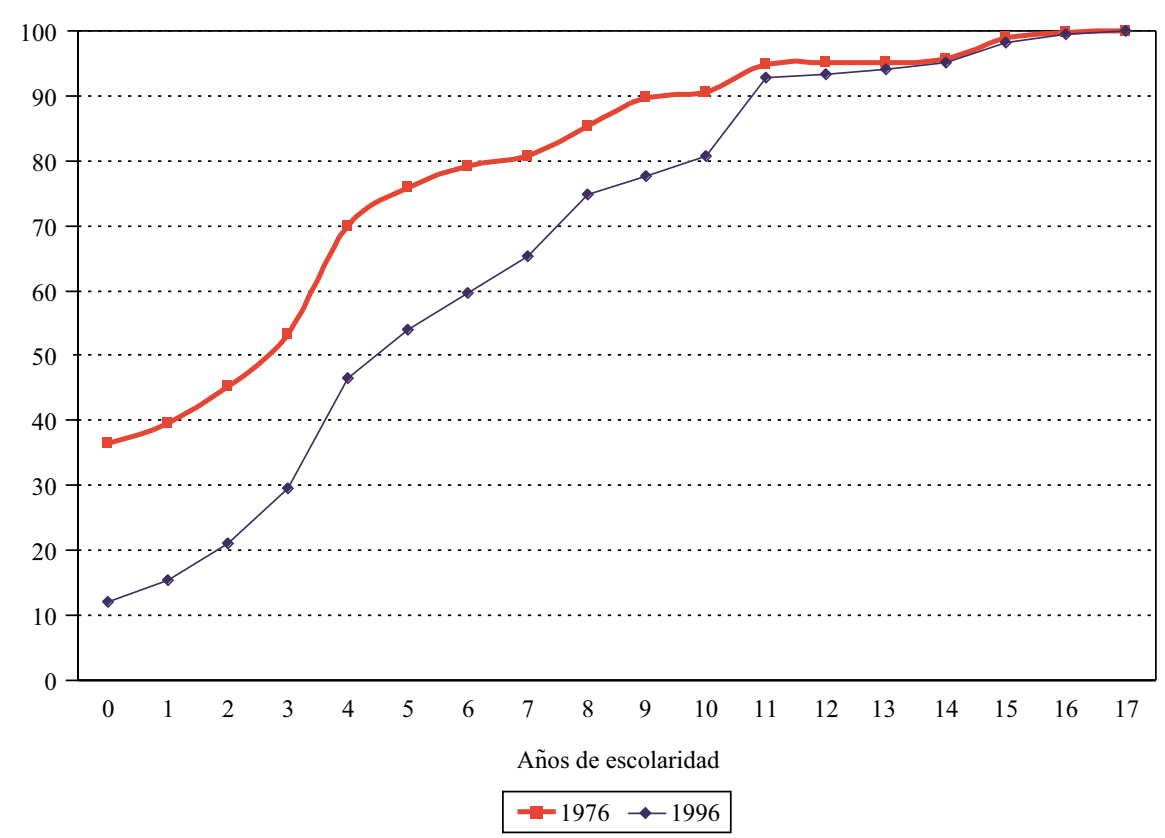


GRAFICO 10

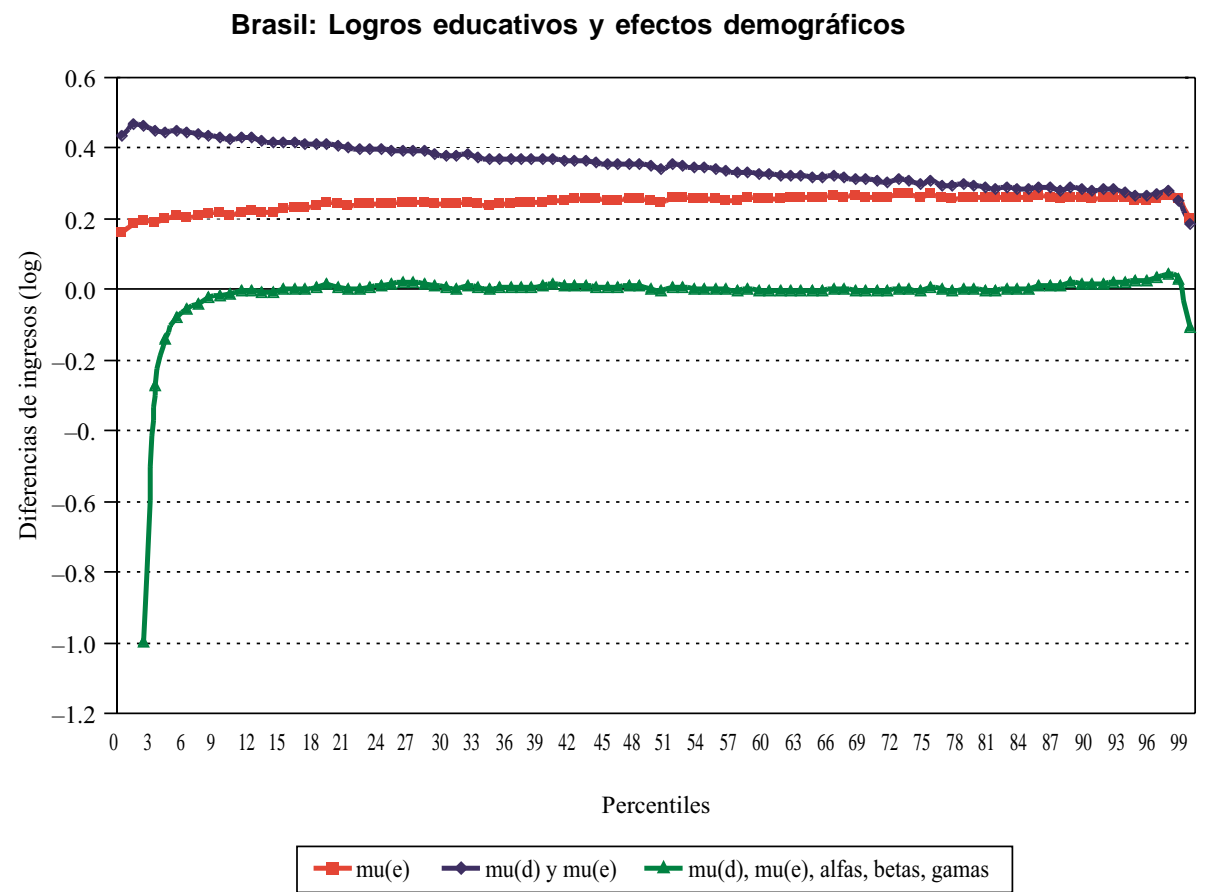

Fuente: IBGE, 1976 y 1996.

GRAFICO 11

\section{Brasil: Una descomposición completa}

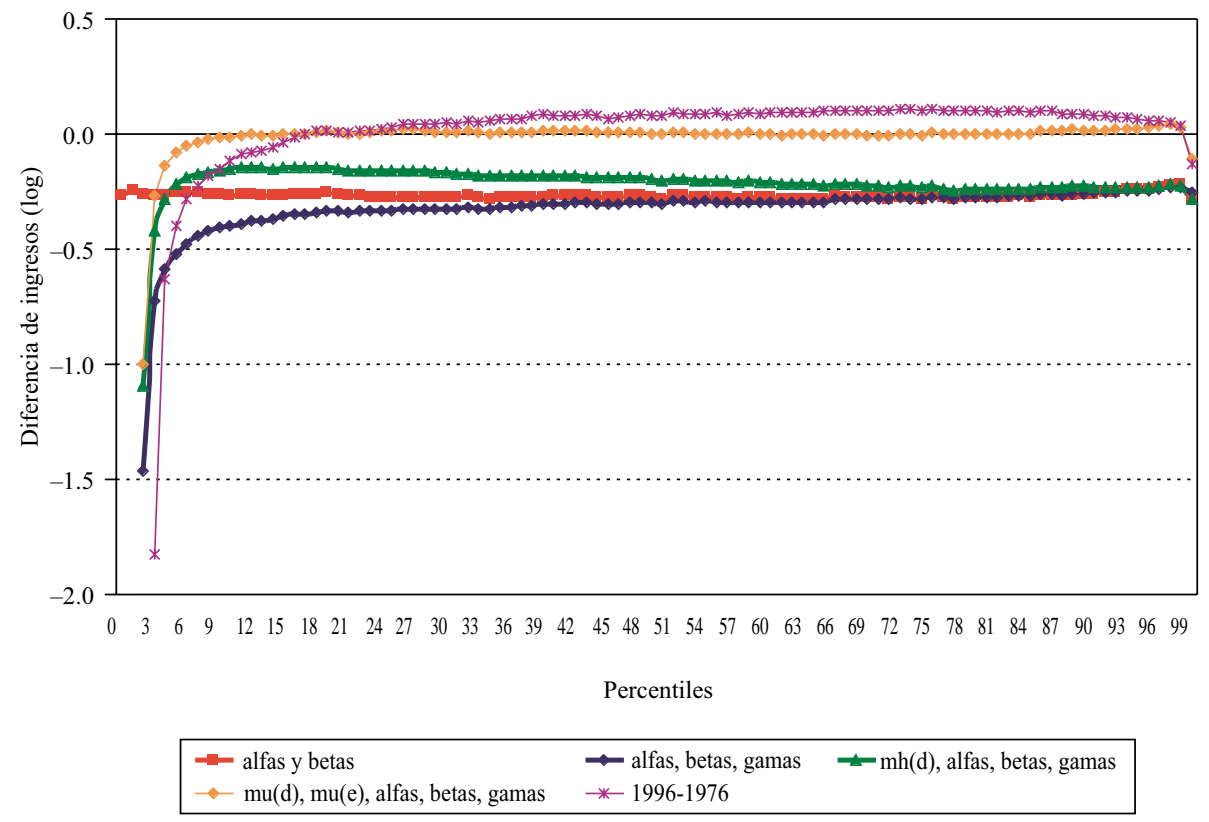

Fuente: IBGE, 1976 y 1996. 
percentil 12 hacia abajo, la simulación sugiere el predominio de efectos nefastos de la opción ocupacional (y, en menor grado, efectos de precio) con grandes pérdidas de ingreso. Se explica así el aumento de la indigencia captado por la línea de pobreza de 30 reales al mes.

La línea inferior en el gráfico 10 representa, en cierto sentido, un ultimo intento, en esta metodología, de simular los distintos cambios que llevaron de la distribución de 1976 a la de 1996. El gráfico 11 es una prueba ilustrativa de este método. La línea "19961976" traza las diferencias en los ingresos efectivos (log) entre las distribuciones observadas de 1996 y de 1976. Junto a ella hemos trazado cada etapa (acumulativa) de nuestras simulaciones. Primero, los efectos empobrecedores (pero casi iguales) de los precios; luego, estos últimos combinados con los efectos altamente

\section{VI}

\section{Conclusiones}

En resumidas cuentas, ¿nos ayuda este ejercicio a comprender mejor la evolución de la distribución del ingreso urbano en el Brasil en este período turbulento de 20 años? Aunque muchos estudiosos tradicionales de la dinámica de la distribución del ingreso podrían haber concluido, a juzgar por los pequeños cambios registrados en el ingreso medio, así como en los índices de desigualdad y los de pobreza, ${ }^{19}$ que había poco - $\mathrm{O}$ nada - que investigar, al adentrarnos un poco más en el problema hemos descubierto un sinnúmero de factores económicos que interactúan para determinar modificaciones importantes en el medio a que hacen frente los individuos y las familias y en sus respuestas a esas modificaciones.

En particular, hemos descubierto que pese a una caída pequeña en la desigualdad cuantificada (aunque las curvas de Lorenz se cruzan) y a un pequeño incremento en el ingreso medio, ha aumentado la pobreza extrema, medida ya sea por líneas bastante bajas de pobreza o por parámetros suficientemente altos de aversión a la pobreza. Este efecto parece derivar de resultados que se relacionan con decisiones de participación y opciones ocupacionales, unidos a una menor

\footnotetext{
${ }^{19}$ Con respecto a la línea de 60 reales al mes, que ya es baja, en una comparación histórica, para el Brasil.
}

empobrecedores de la opción ocupacional; a continuación, la curva que refleja el cuadro un poco menos sombrío surgido de la combinación de estos últimos con los parámetros de las ecuaciones sobre tamaño de la familia. Y, por último, la curva que representa las diferencias entre los ingresos logrados con la simulación en que cambian todos los parámetros y la observada en 1976. Como puede apreciarse, esa última simulación parece ajustarse bastante bien a las diferencias reales. Desde luego que el ejercicio no pretende calcar los cambios reales perfectamente, sino derivar lecciones sobre los efectos diferentes que tienen distintos parámetros y posiblemente sacar algunas conclusiones de política. Sin embargo, el éxito logrado en duplicar los cambios reales con la última simulación da más confianza en la metodología y las lecciones que puede enseñar. rentabilidad en el mercado laboral de la educación y la experiencia. Estos cambios se asocian con un mayor desempleo e informalidad, como cabría esperar, pero éste es un aspecto que merece mayor investigación. Aunque aparentemente hemos identificado a un grupo que está excluido tanto de los mercados del trabajo productivo como de todas las redes importantes de seguridad, no hemos podido interpretar del todo los factores determinantes de sus opciones ocupacionales. Los problemas de la movilidad —agudizados por la medición del indicador de bienestar como ingreso mensual corriente - también requerirán una mayor investigación en este contexto. Las implicaciones de política parecen dirigirse hacia los programas de trabajo focalizados, u otras redes de seguridad, pero sería aventurado entrar en mayores detalles antes de comprender mejor el perfil del grupo que parece haber caído en una situación de extrema pobreza en 1996.

En segundo lugar, hemos descubierto que, incluso por sobre el percentil $15^{\circ}$, donde en general se han estancado los pobres de las zonas urbanas de Brasil, el ascenso fue el resultado de un arduo empeño por avanzar en una pendiente resbaladiza. Tendrán que ganar en promedio dos años más de educación (lo que todavía los deja con un déficit escolar para el nivel de ingreso per cápita del país) y reducir considerablemente su fecundidad a fin de contrarrestar los rendimientos 
decrecientes tanto del mercado formal del trabajo como del trabajo por cuenta propia.

Bien podría ocurrir, como muchos opinan hoy, que la investigación de indicadores no monetarios - como el acceso a servicios o la esperanza de vida al nacerhiciera reconsiderar el calificativo de "década perdida", por ser demasiado duro para el decenio de 1980. Por desgracia, si con estrechez de miras uno se limita a considerar sólo el bienestar medido en términos monetarios, puede decirse que el Brasil urbano no tuvo una década perdida, sino dos.

(Traducido del inglés)

\section{Bibliografía}

Almeida dos Reis, J. G. y R. Paes de Barros (1991): Wage inequality and the distribution of education: A study of the evolution of regional differences in inequality in metropolitan Brazil, Journal of Development Economics, vol. 36, $\mathrm{N}^{\circ} 1$, Amsterdam, Países Bajos, Elsevier Science Publishers, B.V.

Bacha, E. y L. Taylor (1980): Brazilian income distribution in the 1960s: "Facts", model results, and the controversy, L. Taylor, E. Bacha, E. Cardoso y F. Lysy, Models of Growth and Distribution for Brazil, Washington, D.C., Banco Mundial.

Bonelli, R. y G. L. Sedlacek (1989): Distribuição da renda: evolução no último quarto de seculo, G.L. Sedlacek y R. Paes de Barros, Mercado de trabalho e distribuição da renda: uma coletanea, Serie monográfica, $N^{\circ} 35$, Río de Janeiro, Instituto de Planificación Económica y Social (IPEA).

Bourguignon, F., F. Ferreira y N. Lustig (1998): The microeconomics of income distribution dynamics in East Asia and Latin America, World Bank Research Proposal, Washington, D.C., Banco Mundial, abril.

Buhmann, B., L. Rainwater, G. Schmaus y T. Smeeding (1988): Equivalence scales, well-being, inequality and poverty: Sensitivity estimates across ten countries using the Luxembourg income study database, Review of Income and Wealth, vol. 34, $\mathrm{N}^{\circ} 2$, New Haven, Connecticut, International Association for Research in Income and Wealth.

Deaton, A. y C. Paxson (1997): Poverty Among Children and the Elderly in Developing Countries, Working paper, $\mathrm{N}^{\circ} 179$, Princeton, New Jersey, Princeton University, Research Program in Development Studies.

Duryea, S. y M. Szekely (1998): Labor markets in Latin America: A supply-side story, trabajo presentado a la IDB/IIC Annual Meeting, Cartagena de Indias, Colombia, Banco Interamericano de Desarrollo (BID)/Corporación Interamericana de Inversiones (CII).

Ferreira, F. y R. Paes de Barros (1999): The slippery slope: Explaining the increase in extreme poverty in urban Brazil;
1976-1996, The Brazilian Review of Econometrics, vol. 19, $\mathrm{N}^{\circ}$ 2, Río de Janeiro, Sociedade Brasileira de Econometria, noviembre.

Fishlow, A. (1972): Brazilian size distribution of income, The American Economic Review, vol. 62, $\mathrm{N}^{\circ}$ 2, Nashville, Tennessee, American Economic Association.

Foster, J., J. Greer y E. Thorbecke (1984): A class of decomposable poverty measures, Econometrica, vol. 52, $\mathrm{N}^{\circ} 3$, Evanston, Illinois, Econometric Society.

Hoffman, R. (1989): Evolucão da distribuição da renda no Brasil, entre pessoas e entre familias, 1979/86, en G.L. Sedlacek y R. Paes de Barros, Mercado de trabalho e distribuição da renda: uma coletanea, Serie monográfica, $N^{\circ} 35$, Río de Janeiro, IPEA.

IBGE (Instituto Brasileño de Geografía y Estadística) (1976): Pesquisa Nacional por Amostra de Domicilios, Brasilia, Fundação IBGE.

(1981): Pesquisa Nacional por Amostra de Domicilios, Brasilia, Fundação IBGE.

(1985): Pesquisa Nacional por Amostra de Domicilios, Brasilia, Fundação IBGE.

(1996): Pesquisa Nacional por Amostra de Domicilios, Brasilia, Fundação IBGE.

Juhn, C., K. Murphy y B. Pierce (1993): Wage inequality and the rise in returns to skill, Journal of Political Economy, vol. 101, $\mathrm{N}^{\circ} 3$, Chicago, Illinois, The University of Chicago Press.

Langoni, C.G. (1973): Distribuição da renda e desenvolvimento econômico do Brasil, Río de Janeiro, Expressão e Cultura.

Mookherjee, D. y A. F. Shorrocks (1982): A decomposition analysis of the trend in UK income inequality, The Economic Journal, vol. 92, $\mathrm{N}^{\circ} 368$, Oxford, Reino Unido, The Royal Economic Society.

Ramos, L. (1993): A distribuição de rendimentos no Brasil: 1976/ 85, Río de Janeiro, IPEA. 